\title{
The reaction of NADPH with bovine mitochondrial NADH:ubiquinone oxidoreductase revisited
}

\author{
I. Proposed consequences for electron transfer in the enzyme
}

\author{
Simon P. J. Albracht
}

Received: 8 February 2010 / Accepted: 21 June 2010 / Published online: 14 July 2010

(C) The Author(s) 2010. This article is published with open access at Springerlink.com

\begin{abstract}
Bovine NADH:ubiquinone oxidoreductase (Complex I) is the first complex in the mitochondrial respiratory chain. It has long been assumed that it contained only one FMN group. However, as demonstrated in 2003, the intact enzyme contains two FMN groups. The second FMN was proposed to be located in a conserved flavodoxin fold predicted to be present in the PSST subunit. The longknown reaction of Complex I with NADPH differs in many aspects from that with NADH. It was proposed that the second flavin group was specifically involved in the reaction with NADPH. The X-ray structure of the hydrophilic domain of Complex I from Thermus thermophilus (Sazanov and Hinchliffe 2006, Science 311, 1430-1436) disclosed the positions of all redox groups of that enzyme and of the subunits holding them. The PSST subunit indeed contains the predicted flavodoxin fold although it did not contain FMN. Inspired by this structure, the present paper describes a re-evaluation of the enigmatic reactions of the bovine enzyme with NADPH. Published data, as well as new freeze-quench kinetic data presented here, are incompatible with the general opinion that NADPH and NADH react at the same site. Instead, it is proposed that these pyridine nucleotides react at opposite ends of the $90 \AA$ long chain of prosthetic groups in Complex I. Ubiquinone is proposed to react with the Fe-S clusters in the TYKY subunit deep inside the hydrophilic domain. A new model for electron transfer in Complex I is proposed. In the accompanying paper this model is compared with the one advocated in current literature.
\end{abstract}

S. P. J. Albracht ( $\bowtie)$

Swammerdam Institute for Life Sciences,

University of Amsterdam,

Nieuwe Achtergracht 166,

NL-1018 WV Amsterdam, The Netherlands

e-mail: s.p.j.albracht@uva.nl
Keywords NADH:ubiquinone oxidoreductase · Complex I $\cdot$ NADPH $\cdot$ Rapid kinetics $\cdot$ EPR

\section{Introduction}

The experiments in this paper deal solely with bovine-heart NADH:ubiquinone oxidoreductase (EC 1.6.5.3; Complex I) as present in submitochondrial particles (SMP). This complex has a molecular mass of ca. $980 \mathrm{kDa}$, consists of 45 subunits and is located in the mitochondrial inner membrane (Fearnley and Walker 1992; Walker 1992; Hirst et al. 2003; Carroll et al. 2006). A flavin molecule (FMN) accepts the two reducing equivalents from the hydride formed after the heterolytic cleavage of NADH. Subsequently electrons are transferred, one at the time, via a number of EPR-detectable iron-sulphur (Fe-S) clusters (Beinert and Albracht 1982; Ohnishi 1998) to ubiquinone $\left(\mathrm{Q}_{10}\right)$. This process is coupled to the extrusion from the mitochondrial matrix of ca. two protons per electron (Wikström 1984; Galkin et al. 1999). The resulting proton-motive force drives the synthesis of ATP from ADP and inorganic phosphate.

Amino-acid sequence analyses indicated potential binding sites for one FMN, two [2Fe-2S] clusters and six [4Fe4S] clusters (Fearnley and Walker 1992). Bovine Complex I can also react with NADPH at a site considered to be different from the NADH site (Hatefi 1968; Hatefi and Hanstein 1973; Hatefi and Bearden 1976; Albracht 1982; Bakker and Albracht 1986; Van Belzen and Albracht 1989). This has led to the finding that the intact bovine enzyme contains a second FMN group (here termed FMN-a) proposed to be bound to the conserved flavodoxin fold in the PSST subunit (Albracht et al. 2003). This fold was first uncovered by sequence comparisons of the PSST-like 
subunits of Complex I from different origins with the evolutionary-related small subunit of [ $\mathrm{NiFe}]$-hydrogenases, for which the 3D structure was known (Volbeda et al. 1995; Volbeda et al. 1996) (for overviews see (Albracht and De Jong 1997; Albracht and Hedderich 2000; Albracht et al. 2003)). The FMN molecule reacting with NADH (here termed FMN-b) is bound to the $51-\mathrm{kDa}$ subunit (Fearnley and Walker 1992).

The global surface structure of the bovine enzyme has been reconstructed from images of single molecules in vitrified ice using electron cryo-microscopy (Grigorieff 1998). The complex has an L shape, whereby the base of the L (containing the hydrophobic domain) is considered to reside in the mitochondrial inner membrane, whereas the stalk of the $\mathrm{L}$ (containing the hydrophilic domain) sticks out into the matrix space. This has also been found for the mitochondrial enzymes from Neurospora crassa (32 subunits) (Leonard et al. 1987; Hofhaus et al. 1991; Guénebaut et al. 1997) and Yarrowia lipolytica (40 subunits) (Djafarzadeh et al. 2000; Radermacher et al. 2006), and for the bacterial enzymes from Escherichia coli (13 subunits) (Guénebaut et al. 1998; Morgan and Sazanov 2008) and Aquifex aeolicus (14 subunits) (Peng et al. 2003). As expected from the number of subunits, the detailed contours of the stalk and base part of the L-shaped molecule differ among the enzymes (Zickermann et al. 2009; Clason et al. 2010).

In 2006 the three-dimensional structure of the hydrophilic domain of Complex I from Thermus thermophilus has been solved to $3.3 \AA$ resolution (Sazanov and Hinchliffe 2006). It showed one FMN group, two [2Fe-2S] clusters (called N1a and N1b) and seven [4Fe-4S] clusters (N2, N3, $\mathrm{N} 4, \mathrm{~N} 5, \mathrm{~N} 6 \mathrm{a}, \mathrm{N} 6 \mathrm{~b}$ and N7), i.e. one more than predicted for the bovine enzyme (Fearnley and Walker 1992). The predicted flavodoxin fold in the PSST subunit (Albracht and Hedderich 2000) was present, but empty. The binding motif for the cubane cluster N7 in the $75-\mathrm{kDa}$ subunit is absent in the bovine enzyme. The prosthetic groups are approximately positioned along a straight line and span a distance of ca. $90 \AA$, representing an 'electric wire' through the enzyme, nearly perpendicular to the inner membrane. The structure of the NADH-reduced enzyme nicely showed how NADH binds to FMN in the $51-\mathrm{kDa}$ subunit (Berrisford and Sazanov 2009). Subunits homologous to those in the T. thermophilus enzyme are found in Complex I from many organisms. Hence, the structure of this enzyme serves as a template for that of other enzymes. For the bovine enzyme, the structure predicts the presence of $28 \mathrm{Fe}$ atoms per FMN. Surprisingly, the best classical preparations of the bovine-heart enzyme, here referred to as Hatefi's Complex I and Singer's NADH dehydrogenase, contain 16 to 18 non-heme Fe atoms per FMN (Hatefi et al. 1962; Ringler et al. 1963; Cremona and Kearney 1964; Lusty et al. 1965; Orme-Johnson et al. 1974a; Ohnishi et al.
1981; Paech et al. 1981; Kowal et al. 1986) (see accompanying paper for a detailed overview (Albracht 2010)).

Bovine SMP and Hatefi's Complex I (Hatefi et al. 1962) react with NADPH. In SMP the rate of the NADPH $\rightarrow \mathrm{O}_{2}$ reaction at $\mathrm{pH} 7.5$ and $30^{\circ} \mathrm{C}$ amounts to 6 to $50 \mathrm{nmol}$ NADPH.min ${ }^{-1} \cdot \mathrm{mg}^{-1}$ (Rydström et al. 1978; Hatefi 1973; Hatefi and Hanstein 1973) depending on the type of SMP preparation. This is $0.5 \%$ to $5 \%$ of the $\mathrm{NADH} \rightarrow \mathrm{O}_{2}$ reaction rate. The reaction is strongly dependent on $\mathrm{pH}$, being 35 to 40 times faster at $\mathrm{pH} 6$ than at $\mathrm{pH} 9$; the $\mathrm{NADH}$-oxidation rate varies only 2-fold (being maximal at $\mathrm{pH} 7$ ) (Hatefi and Hanstein 1973; Djavadi-Ohaniance and Hatefi 1975; Rydström et al. 1978). It was initially proposed (Rydström et al. 1978) that NADPH reacts only at the NADH-reaction site. The negative charge of the 2'-phosphate group of NADPH $\left(\mathrm{pK}_{\mathrm{a}} 6.1\right.$ (Galante and Hatefi 1979)) was supposed to hinder its reaction at this site at neutral $\mathrm{pH}$. The energylinked transhydrogenase was not involved, since its inactivation by trypsin or butanedione had little effect on the NADH- or NADPH-oxidation reactions. The $\mathrm{K}_{\mathrm{m}}$ values of NADH and NADPH for the oxidation reactions differ by two orders of magnitude ( 7 to $8 \mu \mathrm{M}$ for NADH in SMP and Hatefi's Complex I, and 550 to $570 \mu \mathrm{M}$ for NADPH in SMP (Hatefi et al. 1962; Hatefi and Hanstein 1973; Rydström et al. 1978)). The $\mathrm{K}_{\mathrm{m}}$ value for NADPH in the transhydrogenase reaction is $20 \mu \mathrm{M}$ (Rydström et al. 1971).

Rapid-mixing rapid-freezing experiments showed that all Fe-S clusters from Complex I in SMP are completely reduced within $6 \mathrm{~ms}$ (the dead time of the technique) both at $\mathrm{pH} 8$ and $\mathrm{pH} 6$ and even at $4^{\circ} \mathrm{C}$ (Orme-Johnson et al. 1974a; Van Belzen and Albracht 1989). However, at both $\mathrm{pH}$ values the rate, the pattern and the extent of reduction of the Fe-S clusters by NADPH differ noticeably from that of $\mathrm{NADH}$. The rate of reduction of Fe-S clusters by NADPH is nearly equally fast at $\mathrm{pH} 8$ and $\mathrm{pH} 6$ (reactions completed in 30 to $50 \mathrm{~ms}$ at $22^{\circ} \mathrm{C}$ ) (Bakker and Albracht 1986; Van Belzen and Albracht 1989). This finding did not support the proposal (Rydström et al. 1978) of protonation of the 2'phosphate group of NADPH as cause for the strong $\mathrm{pH}$ dependence of its reaction with SMP. Besides the reports of the groups of Hatefi, Rydström and Albracht referred to above, there are no other papers dealing with the kinetics of the reaction of Complex I with NADPH. The present report provides new results on this topic, indicating the existence of two pH-dependent switch points in Complex I involved in electron transfer from NADPH to the Q-pool. Once activated, the rate of electron transfer at one of these points is apparently proportional to the proton concentration.

Until now the understanding of the reactions with NADPH and the proposed models to explain them were quite unsatisfactorily (Bakker and Albracht 1986; Van Belzen and Albracht 1989; Albracht and De Jong 1997). 
Using the 3D structure of the hydrophilic domain of the $T$. thermophilus Complex I (Sazanov and Hinchliffe 2006) as a template, the present and previous results have been reinterpreted. This leads to a working model where NADH and NADPH react at opposite ends of the $90 \AA$ long peripheral domain. Ubiquinone is proposed to react at the TYKY subunit situated between the two dehydrogenase modules. At neutral $\mathrm{pH}$ the contribution of the NADPH oxidation catalyzed by the NADPH-dehydrogenase module to the overall electron transfer in SMP is negligible. In addition, it is questionable whether this module is involved in the reduction of ubiquinone by NADH.

Other research groups, most of them studying nonbovine systems, concluded that electron transfer from $\mathrm{NADH}$ to $\mathrm{Q}$ proceeds via FMN in the $51-\mathrm{kDa}$ subunit all the way to the Fe-S cluster in the PSST subunit and from there to ubiquinone (Ohnishi 1998; Walker 1992; Friedrich 2001; Yagi and Matsuno-Yagi 2003; Hinchliffe and Sazanov 2005; Sazanov and Hinchliffe 2006; Zickermann et al. 2009). Possible reasons for the discrepancy between that view and the ones presented here are reviewed in the accompanying paper.

\section{Materials and methods}

The experiments in this report have been performed in the E.C. Slater Institute for Biochemical Research at the University of Amsterdam in 1990 by R. van Belzen during his graduate study under supervision of SPJA. They are described in a Ph.D. thesis (Van Belzen 1991).

\section{Materials}

At the time, NADH and NADPH were purchased in the purest form available from Boehringer (Mannheim, F.R.G.). Succinate, MES, MOPS, N,N'-dicyclohexylcarbodi-imide (DCCD) and cytochrome $c$ (horse heart) came from Sigma Chemical Co. (Deisenhofen, F.R.G.). Piericidin A, isolated from Streptomyces mobaraensis (Singer 1979) and dissolved in ethanol, was a gift from Dr. S. de Vries. Coenzyme $\mathrm{Q}_{1}$ was prepared by Mr. A.F. Hartog. All other chemicals were of analytical grade.

\section{Submitochondrial particles}

Submitochondrial particles (SMP) were prepared by sonification of bovine-heart mitochondria. The yield of particles was important because the freeze-quench technique consumes large amounts of material. The time of sonification was therefore increased from 2 min (Löw and Vallin 1963) to four times $2 \mathrm{~min}$ with continuous cooling in an ice-water bath (and intermittent periods of $2 \mathrm{~min}$ to cool the sonifier tip), using a Branson B-12 sonifier operating at $80 \%$ of its maximal output. This led to an increase in temperature of the suspension, sometimes to $30^{\circ} \mathrm{C}$. In turn, this induced the (reversible) de-activation of Complex I in oxidized SMP as described by Kotlyar and Vinogradov (Kotlyar and Vinogradov 1990) (see also (De Jong et al. 1994)). This de-activated state manifests itself as a lag phase (few seconds) in the oxidation of NADH, as first observed with horse-heart SMP (Minakami et al. 1964). Because the extended sonification also resulted in SMP without any respiratory control, a situation not important for the present experiments, a simple sonification medium was used of $0.25 \mathrm{M}$ sucrose and $50 \mathrm{mM}$ Tris- $\mathrm{HCl}$ ( $\mathrm{pH} \mathrm{8.0)}$.

Unless stated otherwise, the particles were treated with butanedione in order to inactivate the transhydrogenase activity (Van Belzen and Albracht 1989). Treatment with DCCD was performed at $30^{\circ} \mathrm{C}$ by adding $400 \mathrm{mM}$ DCCD (dissolved in ethanol) to SMP (40 $\mathrm{mg}$ of protein per $\mathrm{ml}$ ) in $0.25 \mathrm{M}$ sucrose, $100 \mathrm{mM}$ Tris- $\mathrm{HCl}(\mathrm{pH} \mathrm{8.0)}$ to the desired final DCCD concentration. At regular intervals samples were drawn for activity measurements. The incubation was ended by diluting the particles to $5 \mathrm{mg}$ of protein per $\mathrm{ml}$ by adding ice-cold buffer after which the particles were centrifuged for $30 \mathrm{~min}$ at 100,000 $\mathrm{g}$ and resuspended in a buffer without DCCD. Suspensions of SMP with a low buffering capacity were prepared by re-suspending centrifuged particles in $0.25 \mathrm{M}$ sucrose, $1 \mathrm{mM}$ butanedione ( $\mathrm{pH} 8.0$ ).

\section{Activity assays}

Assays for the oxidation activities of NADH, NADPH and succinate were carried out polarographically at $30^{\circ} \mathrm{C}$ as has been described (Van Belzen and Albracht 1989). The titrations of NAD(P)H oxidation activities with the inhibitor piericidin A were carried out as in (Van Belzen et al. 1990). The $\mathrm{NADH} \rightarrow \mathrm{Q}_{1}$ reductase assay was carried out at $30^{\circ} \mathrm{C}$ in $0.25 \mathrm{M}$ sucrose, $100 \mathrm{mM}$ Tris- $\mathrm{HCl}, 2 \mathrm{mM}$ $\mathrm{KCN}\left(\mathrm{pH} 8.0\right.$ ) containing $75 \mu \mathrm{M} \mathrm{Q}_{1}$ and $0.5 \mathrm{mg}$ of protein per $\mathrm{ml}$. The decrease of the absorption at $340 \mathrm{~nm}$ was monitored after addition of $60 \mu \mathrm{M}$ NADH. The $\mathrm{NADH} \rightarrow$ cytochrome $c$ reductase assay was carried out at $30^{\circ} \mathrm{C}$ in the same buffer containing $40 \mu \mathrm{M}$ cytochrome $c$ instead of $Q_{1}$ and $0.1 \mathrm{mg}$ of protein per $\mathrm{ml}$. In this case the increase of the absorption at $550 \mathrm{~nm}$ was monitored after addition of $60 \mu \mathrm{M}$ NADH. Protein concentrations were determined by the biuret method (Gornall et al. 1949; Cleland and Slater 1953).

Freeze-quench experiments

Rapid-mixing rapid-freezing experiments were performed at room temperature $\left(22^{\circ} \mathrm{C}\right)$ as described (Van Belzen and Albracht 1989). Mixing in a mixing chamber was always 
on a 1:1 (v/v) basis. Concentrated SMP suspensions (60$80 \mathrm{mg} / \mathrm{ml}$ ) were mixed with $10 \mathrm{mM}$ NADPH in buffers indicated in the figures. Maximal development of the signals was induced by mixing with $10 \mathrm{mM} \mathrm{NADH}$; in this case equally strong signals were obtained in the $\mathrm{pH}$ range 5.5 to 8.3. In order to prevent formation of air bubbles, the SMP suspensions were warmed to room temperature (and air bubbles were removed by short centrifugation at $1000 \times \mathrm{g}$ ) before filling the syringe. After that, it usually took at least $15 \mathrm{~min}$ before the first reaction could be started. This procedure promoted the further reversible de-activation of Complex I.

\section{EPR spectrometry}

EPR spectrometry and determination of spin concentrations were performed as described (Albracht 1980). The various signals were recorded as in previous studies (Bakker and Albracht 1986; Van Belzen and Albracht 1989) with the following sample temperatures and microwave powers incident to the cavity. For the N1b signal, $45 \mathrm{~K}$ and $2 \mathrm{~mW}$; for the $\mathrm{N} 2$ signal, $17 \mathrm{~K}$ and $0.2 \mathrm{~mW}$; for the $\mathrm{N} 3$ and $\mathrm{N} 4$ signals, $9 \mathrm{~K}$ and $20 \mathrm{~mW}$. The weak $\mathrm{N} 3$ and $\mathrm{N} 4$ signals had to be recorded under slightly saturating conditions in order to obtain an acceptable signal-to-noise ratio. The amplitudes of the 1.94, 1.92, 1.88 and 1.86 lines were determined (see Fig. 1) relative to those obtained after optimal reduction by NADH. No changes in the lineshapes of the EPR spectra could be detected after DCCD treatment.

\section{Results}

In the present paper the term 'Complex I' is used for the enzyme in membrane preparations and for purified preparations that catalyze an inhibitor-sensitive (rotenone or piericidin A) $\mathrm{NADH} \rightarrow$ quinone activity without any reconstitution efforts. Preparations without this activity are called 'NADH dehydrogenase', even although inhibitor-sensitive quinone reductase activity can often be induced by treatment with phospholipids.

Nomenclature of the Fe-S clusters and of the EPR signals from Complex I

These names have changed over time (for overview see (Ohnishi 1998)). Presently, the two binuclear clusters are called N1a and N1b, while the cubane clusters are named N2, N3, N4, N5, N6a, N6b and N7 (Sazanov and Hinchliffe 2006). Cluster N7 is absent in eukaryotic enzymes. In the bovine enzyme cluster N1a cannot be reduced by NADH (Ohnishi 1975).

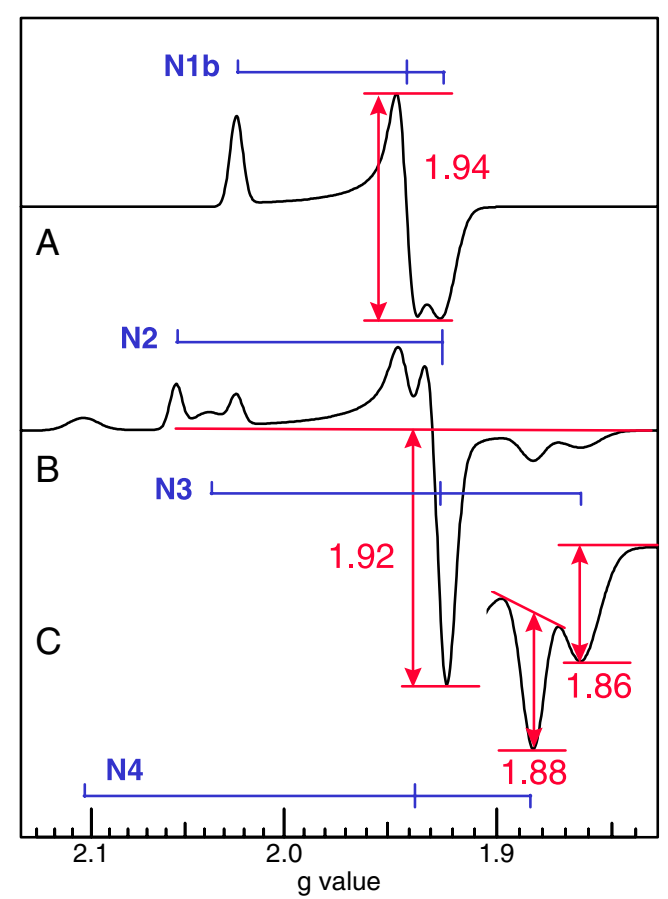

Fig. 1 Lineshapes of the four apparent EPR signals from $\mathrm{NADH}$ reduced bovine-heart Complex I (Hatefi's Complex I). A Lineshape of signal N1b as observed at $45 \mathrm{~K}$. B Overall lineshape as observed at $9 \mathrm{~K}$ under non-saturating conditions. $\mathbf{C}$ Enlarged lineshape of the 1.88 and 1.86 lines. The positions of the $g$ values of each signal are indicated with blue lines. The parameters for simulation of the lineshapes (Albracht et al. 1977; Hearshen et al. 1981) were as follows: for the N1b signal, $g_{1,2,3}=2.023,1.941,1.924$ and widths $(1,2,3)=1.05,1.25,2.1 \mathrm{mT}$; for the N2 signal, $g_{1,2,3}=2.0538,1.925$, 1.925 and widths $(1,2,3)=1.0,1.6,1.6 \mathrm{mT}$; for the N3 signal, $g_{1,2,3}=$ $2.037,1.9263,1.863$ and widths $(1,2,3)=2.5,1.4,3.0 \mathrm{mT}$; for the $\mathrm{N} 4$ signal, $g_{1,2,3}=2.103,1.938,1.884$ and widths $(1,2,3)=2.4,1.9,1.9 \mathrm{mT}$. In spectrum $\mathbf{B}$ the relative intensity of the N1b signal was taken half that of the other signals, this in accordance with extensive determinations of the relative spin concentration of the N1b signal (Albracht et al. 1977, 1979; Van Belzen et al. 1992). The red vertical arrows indicate how the redox behaviour of the four signals is measured via the amplitudes of their main lines at $1.94(\mathrm{~N} 1 b), 1.92(\mathrm{~N} 2), 1.88$ (N4) and $1.86(\mathrm{~N} 3)$. Note that these arrows solely indicate the amplitudes of the several lines and not the position of the relevant $g$ values. Experimental EPR spectra obtained with freeze-quench experiments have been published before and can be found in e.g. (Bakker and Albracht 1986) for the 1.94, 1.88 and 1.86 lines and in Fig. 7 of (De Jong et al. 1994) for the 1.92 line. For normal high-quality experimental lineshapes see e.g. (Albracht et al. 1977; Hearshen et al. 1981; Beinert and Albracht 1982)

The EPR spectrum of the NADH-reduced bovine enzyme is made up of four main signals, the signals N1b, $\mathrm{N} 2, \mathrm{~N} 3$ and N4. A fifth minor signal (N5), first detected in the bovine enzyme (Albracht 1974; Ohnishi 1975; Beinert and Albracht 1982) and later in the enzyme from Paracoccus denitrificans (Yano et al. 2003), is not detected in the yeasts Candida utilis (Albracht and Subramanian 1977), Pichia angusta and Pichia pastoris (Bridges et al. 2009). The assignment of the four main signals to the seven Fe-S clusters in the bovine enzyme is still a matter of debate. A 
schematic overview of the EPR signals, and the method used in the present and previous papers to monitor their kinetic behaviour, is displayed in Fig. 1.

Recently, the group of Hirst proposed a nomenclature for the clusters based on their nuclearity ( $2 \mathrm{Fe}$ or $4 \mathrm{Fe}$ ), the subunit location (using the E. coli designation) and Cysligand composition ( $\mathrm{C}$ for four $\mathrm{Cys}$, and $\mathrm{H}$ for three Cys and one His ligand) (Yakovlev et al. 2007). In this notation the clusters N1a and N1b were called $2 \mathrm{Fe}[\mathrm{E}]$ and $2 \mathrm{Fe}[\mathrm{G}]$, respectively. One year later yet another nomenclature, based on the subunit designation of the bovine enzyme, led to designations as $2 \mathrm{Fe}[24]$ and $2 \mathrm{Fe}[75]$ for the clusters N1a and N1b, respectively (Reda et al. 2008). For simplicity, the present paper will refer to the eight clusters as clusters $a$ to $h$. Table 1 summarises the cluster designations and Fig. 2 shows the clusters $a$ to $h$ in the $T$. thermophilus structure. Figure 2 also includes details about the proposals based on the experiments described below. Note that the proposed reaction sites of NADH and $\mathrm{NADPH}$ are at opposite ends of the chain of redox groups and that $\mathrm{Q}_{10}$ is proposed to react deep inside the hydrophilic domain.

Uncertainties in the assignments of $g$-value sets to the four EPR signals

In the Amsterdam group the total spin concentration represented by the EPR spectrum of Hatefi's Complex I reduced with NADH was found to be seven times the spin concentration of the signal of cluster N1b (Albracht et al.

Table 1 Nomenclature of the prosthetic groups in bovine Complex I-The designation used in the present paper is in column 1 (see Fig. 2 for spatial overview). The location of FMN-a has not yet been confirmed experimentally. Column 4 gives the names used in the $T$. thermophilus structure (Sazanov and Hinchliffe 2006)

\begin{tabular}{llll}
\hline 1 Name & 2 Group & 3 Subunit & 4 Name \\
\hline FMN-b & FMN & $51 \mathrm{kDa}$ & FMN \\
FMN-a & FMN & PSST & - \\
$a$ & {$[2 \mathrm{Fe}-2 \mathrm{~S}]$} & $24 \mathrm{kDa}$ & $\mathrm{N} 1 \mathrm{a}$ \\
$b$ & {$[4 \mathrm{Fe}-4 \mathrm{~S}]$} & $51 \mathrm{kDa}$ & $\mathrm{N} 3$ \\
$c$ & {$[2 \mathrm{Fe}-2 \mathrm{~S}]$} & $75 \mathrm{kDa}$ & $\mathrm{N} 1 \mathrm{~b}$ \\
$d$ & {$[4 \mathrm{Fe}-4 \mathrm{~S}]^{\mathrm{p}}$} & $75 \mathrm{kDa}$ & $\mathrm{N} 4$ \\
$e$ & {$[4 \mathrm{Fe}-4 \mathrm{~S}]^{\mathrm{q}}$} & $75 \mathrm{kDa}$ & $\mathrm{N} 5$ \\
$f$ & {$[4 \mathrm{Fe}-4 \mathrm{~S}]^{\mathrm{r}}$} & $\mathrm{TYKY}$ & $\mathrm{N} 6 \mathrm{a}$ \\
$g$ & {$[4 \mathrm{Fe}-4 \mathrm{~S}]^{\mathrm{s}}$} & $\mathrm{TYKY}$ & $\mathrm{N} 6 \mathrm{~b}$ \\
$h$ & {$[4 \mathrm{Fe}-4 \mathrm{~S}]$} & PSST & $\mathrm{N} 2$ \\
\hline
\end{tabular}

Superscripts: ${ }^{\mathrm{p}}$, cluster with four Cys ligands; ${ }^{\mathrm{q}}$, cluster with three Cys ligands and one His ligand; ${ }^{\mathrm{r}}$, cluster in the TYKY subunit closest to the $75-\mathrm{kDa}$ subunit; ${ }^{\mathrm{s}}$, cluster in the TYKY subunit closest to the PSST subunit.

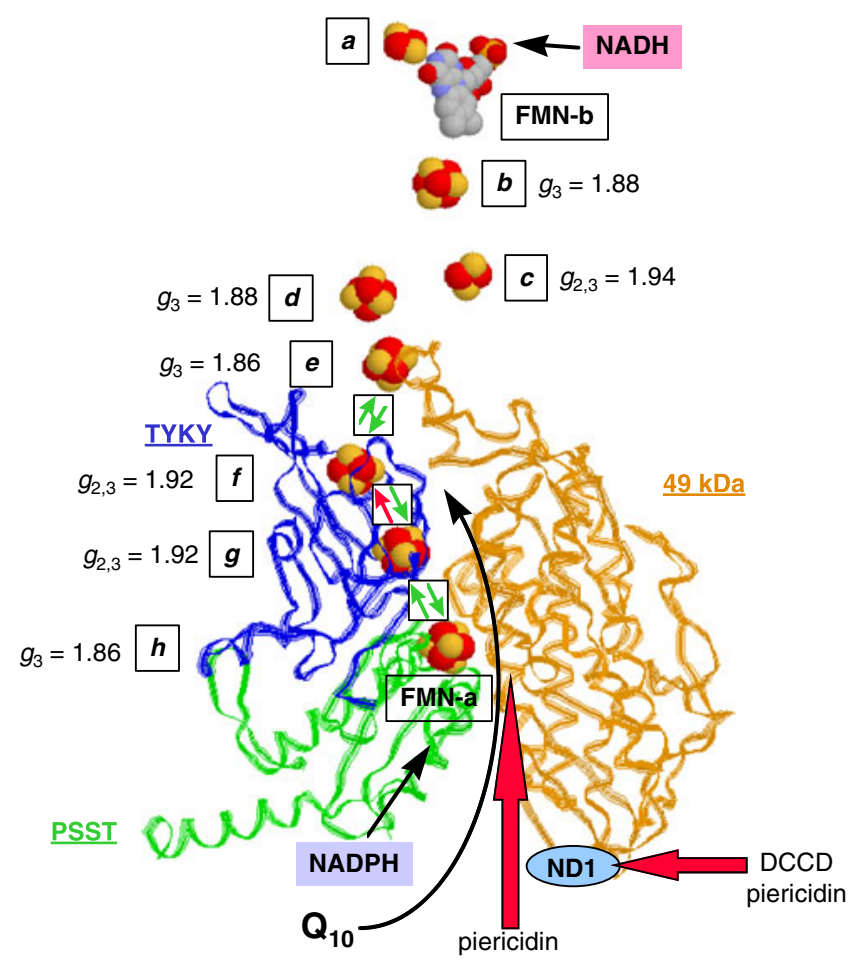

Fig. 2 Working model of the reactions of bovine-heart Complex I with $\mathrm{NADH}$ and NADPH, and assignment of EPR signals to the Fe-S clusters as proposed in this paper, using the structure of the hydrophilic domain of the enzyme from T. thermophilus (PDB entry 2fug (Sazanov and Hinchliffe 2006)) as a template. Cluster N7 is omitted, as this is absent in the bovine enzyme. NADH reacts at FMN$\mathrm{b}$ and further electron transfer proceeds via clusters $b, c$ and $d$. NADPH delivers its hydride at FMN-a, which is proposed to be bound to the conserved flavodoxin fold in the PSST subunit. At $\mathrm{pH} 8$ electrons from FMN-a flow rapidly into the Fe-S clusters $h$ (PSST) and $g$ (TYKY), but not any further (thin red arrow) and they cannot reach $\mathrm{Q}_{10}$. A pH of 6 unlocks electron transfer (at the position of the thin red arrow) to the clusters $f$ (TYKY) and $e(75 \mathrm{kDa})$ and to ubiquinone. A wide hydrophobic cleft allows $\mathrm{Q}_{10}$ (Sazanov and Hinchliffe 2006) (and piericidin A) to enter the enzyme. It is proposed here that $\mathrm{Q}_{10}$ reacts close to cluster $f$. Rotenone and piericidin A can bind to the hydrophobic ND1 subunit as well as close to a site in the 49-kDa subunit, the site structurally equivalent to that of the Ni-Fe site in $[\mathrm{NiFe}]$-hydrogenases (thick red arrows). $N, N^{\prime}$-dicyclohexylcarbodiimide (DCCD) binds specifically to the ND1 subunit. The position and size of the ND1 subunit are arbitrary. Thin green arrows indicate fast electrons transfer between the Fe-S clusters when electrons enter via FMN-a. See text for further details

1977; Albracht et al. 1979; Van Belzen et al. 1992; Finel et al. 1994; Albracht and De Jong 1997). This means that the spectrum receives contributions from one [2Fe-2S] cluster (N1b) and six [4Fe-4S] clusters; thus all clusters, except N1a, are EPR detectable. Kinetic studies with NADPH showed that the 1.92 line (from signal N2), the 1.88 line (from signal N4) and the 1.86 line (from signal N3) each receive contributions from two kinetically-distinct [4Fe-4S] clusters (Albracht 1982; Bakker and Albracht 1986; Albracht and Bakker 1986; Van Belzen and Albracht 1989). The presence in the EPR spectrum of signals from 
one $2 \mathrm{Fe}$ cluster and six $4 \mathrm{Fe}$ clusters creates some uncertainties in previously used $g$-value assignments. The original assignment was based on the assumption that each of the four apparent EPR signals (N1b, N2, N3 and N4) represented a specific Fe-S cluster (likewise called N1b, $\mathrm{N} 2$, N3 and N4). For two signals, the N1b and N2 signals, the assignment of $g$ values (indicated as blue lines in Fig. 1) is straightforward because these signals can be easily monitored without much interference of other signals (see e.g. (Ohnishi 1975; Albracht et al. 1977; Beinert and Albracht 1982; Ohnishi 1998)). As, according to repeated measurements in the Amsterdam group, the N2 signal in the bovine enzyme represents a spin concentration twice that of the N1b signal (Albracht et al. 1977; Albracht et al. 1979; Van Belzen et al. 1992), it follows that it is caused by two clusters with (at X-band and even at Q-band (Albracht 1974; Albracht 1984)) indistinguishable EPR signals.

The N3 and N4 signals each have an intensity equal to that of the N2 signal (Beinert and Albracht 1982; Ohnishi 1998). Hence also these signals each represent two spins per Complex I. Thus, the 1.88 line as well as the 1.86 line each receive contributions from two different clusters. Assignment of proper $g$-value sets to the rhombic and broad N3 and N4 signals is difficult because they are nearly always overlapped by the much sharper N1b and N2 signals (Fig. 1). The N3 and N4 signals can be monitored by two apparent $g_{1}\left(g_{\mathrm{z}}\right)$ lines (one at 2.10 and the other at 2.04) and two $g_{3}\left(g_{\mathrm{x}}\right)$ lines (one at 1.88 and the other at 1.86). The $g_{2}$ $\left(g_{\mathrm{y}}\right)$ lines in the $g=1.93$ to 1.94 region are masked by overlap of the much more intense lines of the N1b and N2 signals. In theory, the lineshape of the combined N3 and N4 signals, representing four different Fe-S clusters, can be obtained by eight different combinations of the two $g_{1}$, two $g_{2}$ and two $g_{3}$ values.

Experimental indications for assigning the $g_{1}$ line at 2.10 to two different $\mathrm{Fe}-\mathrm{S}$ cluster with different $g_{3}$ lines and quite different midpoint potentials have been reported in the 1970s. In Hatefi's Complex I, one cluster, with a relatively high potential, was assigned to have $g_{1}=2.100$ and $g_{3}=$ 1.886 , while another one, with a relatively low potential, was thought to have $g_{1}=2.103$ and $g_{3}=1.862$ (OrmeJohnson et al. 1971; Orme-Johnson et al. 1974a; Beinert and Albracht 1982). Also in redox titrations with pigeonheart SMP it was found that the 2.1 line received contributions from two clusters with quite different midpoint potentials $(-240 \mathrm{mV}$ and $-410 \mathrm{mV})$ and slightly different $g_{3}$ lines (Ohnishi 1975). Together these findings imply that also the $g_{1}=2.037$ line, which was recognized later (Albracht et al. 1977; Ohnishi 1979), has contributions from two clusters with different $g_{3}$ lines, one with $g_{3}=1.88$ and the other with $g_{3}=1.86$.

Simulations based on these possibilities show that the amplitudes of the 1.88 or 1.86 lines change by at most $15 \%$ when the $g_{1}$ value in a $g$-value set is shifted from 2.10 to 2.037 (not shown). So, in theory the relative contributions of the two individual signals to the amplitude of the 1.88 line may deviate from a strict 50/50 distribution; the same holds for the 1.86 line. However, in experimental spectra of these broad signals, in particular in the rather noisy spectra from freeze-quench experiments with SMP (Bakker and Albracht 1986), such small effects will be difficult to detect. Thus, such simulations cannot be used to distinguish between the eight possible combinations of $g$ values mentioned above. Instead, the N3 and N4 lineshapes simulated with the conventional two sets of $g$ values (as used for trace B of Fig. 1) are sufficient to describe the main signals.

The $\mathrm{pH}$ dependence of the $\mathrm{NAD}(\mathrm{P}) \mathrm{H} \rightarrow \mathrm{O}_{2}$ reactions

Figure 3a shows the steady-state rates of oxidation by $\mathrm{O}_{2}$ of NADH and NADPH catalyzed by butanedione-treated SMP at different $\mathrm{pH}$ values. The butanedione treatment inhibits the transhydrogenase reaction by $99 \%$ (Djavadi-Ohaniance and Hatefi 1975; Van Belzen and Albracht 1989). At $\mathrm{pH} 5.5$ the rates of oxidation of NADH and NADPH are equal. The experiments confirm the observation that the large increase of the NADPH oxidation reaction from $\mathrm{pH} 8.5$ to $\mathrm{pH} 5.7$ is not due to the action of transhydrogenase (Djavadi-Ohaniance and Hatefi 1975; Rydström et al. 1978). It is in agreement with the notion that Complex I is directly involved in the rotenone- and piericidin-A-sensitive NADPH oxidation.

To visualise the results of the freeze-quench experiments in this paper, the structure in Fig. 2 is reduced to the modular one shown in Fig. 3b. It was earlier established that at $\mathrm{pH} 8$ only $50 \%$ of the 1.92 line in bovine SMP could be induced by NADPH; this level was reached in $50 \mathrm{~ms}$ and did not change at longer times. The reaction was not affected by the presence of rotenone, the absence of $\mathrm{O}_{2}$ or the absence of $\mathrm{Q}_{10}$ (Van Belzen and Albracht 1989). Also at pH 6.2 NADPH induced $50 \%$ of the 1.92 and 1.86 lines. However, in this case the presence rotenone or the absence of $\mathrm{Q}_{10}$ resulted in the appearance of $80 \%$ to $100 \%$ of the lines within $30 \mathrm{~ms}$. The 1.94 line $(\mathrm{N} 1 \mathrm{~b}$, cluster $c)$ remained always less than $50 \%$ of its NADH-induced amplitude (Bakker and Albracht 1986; Albracht and Bakker 1986; Van Belzen and Albracht 1989). To further investigate the effects of $\mathrm{pH}$, the following experiments were performed.

Reduction of SMP by NADPH at $\mathrm{pH} 8$ and the effects of a subsequent $\mathrm{pH}$ jump from 8 to 6.2

For this purpose a three-syringe experiment was performed. First, SMP (syringe 1) were mixed with NADPH (syringe 2 ), both in aerobic media at $\mathrm{pH} 8.0$ with a low buffering 

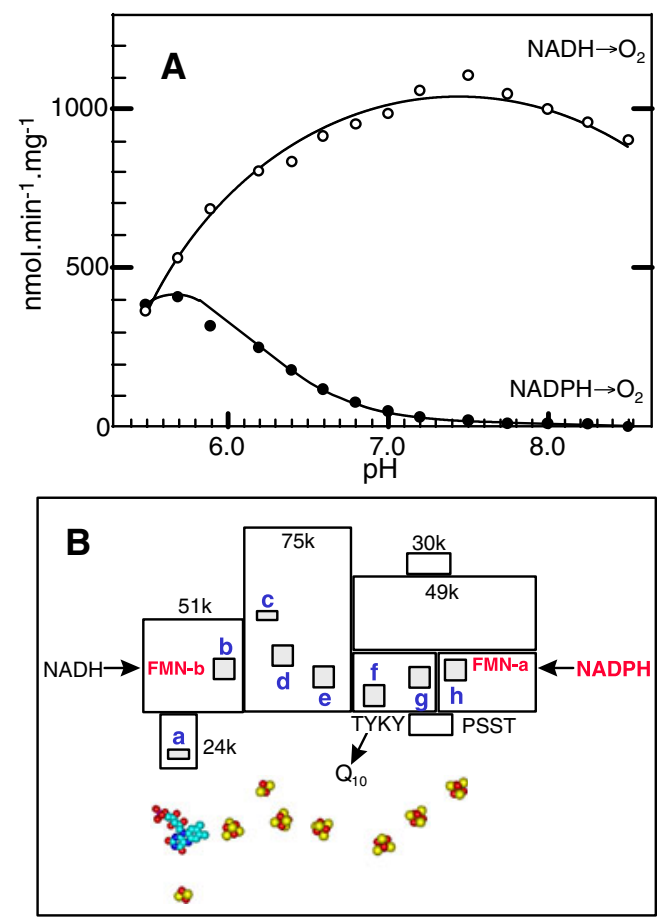

Fig. 3 The $p H$ dependencies of the NADH-and NADPH-oxidation activities catalyzed by butanedione-treated $S M P$ at $30^{\circ} \mathrm{C}$. A Open circles, oxidation activities with $5 \mathrm{mM} \mathrm{NADH}$; filled circles, oxidation activities with $5 \mathrm{mM}$ NADPH. The reaction medium consisted of $0.25 \mathrm{M}$ sucrose, $1 \mathrm{mM}$ butanedione and various buffers: $100 \mathrm{mM}$ MES-KOH (pH 5.5 to 6.6), $100 \mathrm{mM}$ MOPS-KOH (pH 6.8 to 7.75) or $100 \mathrm{mM}$ Tris- $\mathrm{HCl}$ (pH 7.75 to 8.5). B Modular representation of seven of the hydrophilic subunits of bovine Complex I and their prosthetic groups. The relative positions of the groups are based on those of the prosthetic groups in the hydrophilic domain of the T. thermophilus enzyme depicted below the Complex I module (co-ordinates taken from PDB entry 2 fug (Sazanov and Hinchliffe 2006)). An extra flavin, FMN-a, is placed in the PSST subunit. The reaction site of $\mathrm{Q}_{10}$ is proposed to be close to cluster $f$ in the TYKY subunit. In the block scheme, the clusters $a$ and $c$ (rectangles) are [2Fe-2S] clusters, while the other ones (squares) are [4Fe-4S] clusters. Note that the $30-\mathrm{kDa}$ subunit clamps the $49-\mathrm{kDa}$ and TYKY subunits together

capacity, and the reaction was allowed to continue for $1000 \mathrm{~ms}$. Then a concentrated buffer of $\mathrm{pH} 6.2$ was introduced from a third syringe and the reaction was followed in time (Fig. 4a). The reaction with NADPH at $\mathrm{pH} 8.0$ resulted in the appearance of ca. $40 \%$ of the 1.92 and 1.86 lines and only $20 \%$ of the 1.94 and 1.88 lines. As mentioned above, this level of reduction is the same as obtained with NADPH under anaerobic conditions or in $\mathrm{Q}_{10}$-depleted SMP and it is not affected by rotenone (Van Belzen and Albracht 1989). When the $\mathrm{pH}$ was changed (at time zero in the plot) the 1.92 and 1.86 lines fully developed in ca. $280 \mathrm{~ms}$ and then decreased to $50 \%$ after $800 \mathrm{~ms}$. The 1.88 line increased from $20 \%$ to $70 \%$ and then decreased to $40 \%$ of its maximal amplitude. The 1.94 line increased somewhat in the first $100 \mathrm{~ms}$ and then remained nearly constant.
The maximal 1.92 and 1.86 lines, as obtained with NADH, each represent 2 spins per Complex I. This means that at $\mathrm{pH} 8 \mathrm{NADPH}$ reduces approximately two clusters
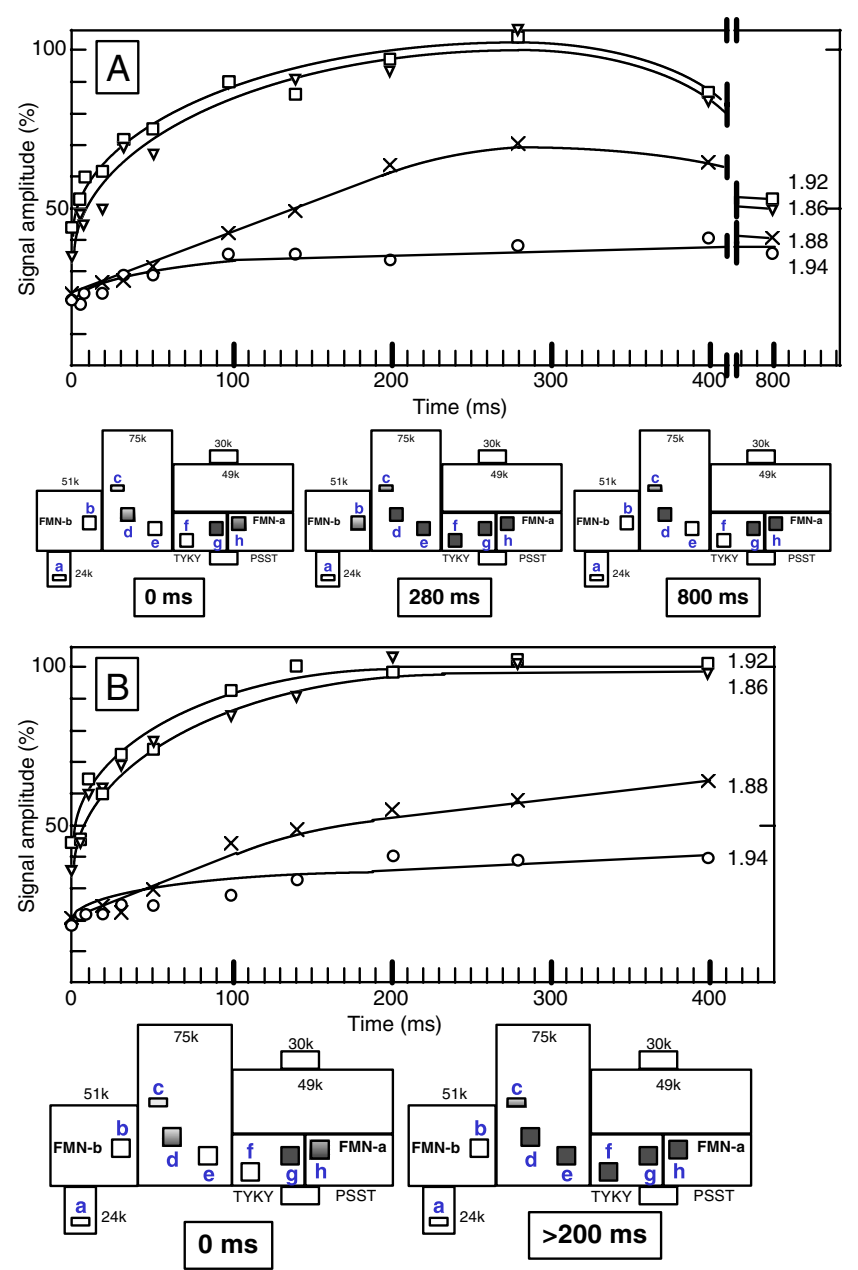

Fig. 4 Reduction of Complex I in SMP by NADPH at $\mathrm{pH} 8$ and the effects of a subsequent $p H$ jump from 8 to 6.2. A Three-syringe experiment in which syringe 1 contained SMP suspended in $0.25 \mathrm{M}$ sucrose, $1 \mathrm{mM}$ butanedione ( $\mathrm{pH} 8.0$ ), syringe 2 held $10 \mathrm{mM} \mathrm{NADPH}$, $0.25 \mathrm{M}$ sucrose ( $\mathrm{pH} 8.0$ ), and syringe 3 was filled with $0.25 \mathrm{M}$ sucrose, $300 \mathrm{mM}$ MES-KOH (pH 6.2). All syringes started to move at the same time. The tubing connecting mixing chamber I with mixing chamber II was calibrated for a reaction time of $1000 \mathrm{~ms}$. The moment of the $\mathrm{pH}$ jump was chosen as the zero time in the figure. The maximal obtainable signal amplitudes were determined by replacing NADPH by NADH in syringe 2 and choosing a reaction time of $29 \mathrm{~ms}$ after the $\mathrm{pH}$ jump. The block schemes below the figure represent the interpretation of the results. Left, redox states at the start of the $\mathrm{pH}$ jump, i.e. after a reaction of the particles with NADPH at pH 8.0 for $1000 \mathrm{~ms}$. Centre, the redox states $280 \mathrm{~ms}$ after the $\mathrm{pH}$ jump. Right, the redox states $800 \mathrm{~ms}$ after the $\mathrm{pH}$ jump. B The same experiment, except for the fact that syringe 1 also contained $45 \mu \mathrm{M}$ rotenone. The block schemes indicate the interpretation of the results. Left: redox states at the start of the $\mathrm{pH}$ jump. Right: redox states from $200 \mathrm{~ms}$ onwards. Plotted are the amplitudes of the 1.94 line (circles), the 1.92 line (squares), the 1.88 line (x signs) and the 1.86 line (triangles), measured as indicated in Fig. 1. In the block schemes, the clusters $a$ and $c$ (rectangles) are $[2 \mathrm{Fe}-2 \mathrm{~S}]$ clusters, while the other ones (squares) are [4Fe-4S] clusters. Open symbols: oxidized clusters; filled symbols: reduced clusters; shaded symbols: cluster population partly reduced 
per Complex I, one with a 1.92 line and the other with a 1.86 line. This level of reduction is reached within $50 \mathrm{~ms}$ (Bakker and Albracht 1986; Van Belzen and Albracht 1989). Figure 4a shows that this reduction level remains unaltered up to $1000 \mathrm{~ms}$. It has been demonstrated by point mutations of conserved Cys residues that both clusters in the TYKY subunit of Rhodobacter capsulatus Complex I in plasma membranes reduced with NADH display an EPR signal with $g_{2,3}=1.92$ (Chevallet et al. 2003). Because clusters with a 1.88 line or a 1.94 line (cluster N1b) were only reduced to $20 \%$ (Fig. $4 \mathrm{a}$ ) it is concluded that NADPH does not react at the NADH site. Instead, it is proposed that in the bovine enzyme at $\mathrm{pH} 8 \mathrm{NADPH}$ reacts with FMN-a and that the clusters $h$ (representing half of the 1.86 line) and $f$ (representing half or the 1.92 line) are reduced (see block scheme in Fig. 4a). Figure 3a shows that under steady-state conditions, i.e. with SMP containing active Complex I, there is nearly no electron transfer from $\mathrm{NADPH}$ to $\mathrm{O}_{2}$ at $\mathrm{pH} 8$.

Although Complex I in the SMP used in Fig. 4a was in the de-activated state (see Materials and methods), the reduction by NADH of all Fe-S clusters was completed within $6 \mathrm{~ms}$, just as in SMP with active Complex I (De Jong and Albracht 1994). This is in agreement with the proposal (Kotlyar and Vinogradov 1990) that in the deactivated enzyme the reoxidation of the NADH-reduced Complex $I$ by $\mathrm{Q}_{10}$ is not functional, leading to a lag time in the $\mathrm{NADH} \rightarrow \mathrm{Q}_{10}$ activity as first observed in 1964 (Minakami et al. 1964). With this in mind, it is proposed that the increase and subsequent decrease of the 1.92 and 1.86 lines in Fig. 4a were caused by activation phenomena in the NADPH-reduced Complex I, induced by the change of $\mathrm{pH}$ from 8.0 to 6.2 (see block schemes in Fig. 4a). Apparently, the low $\mathrm{pH}$ first unlocked electron transfer from cluster $g$ to cluster $f$ in the TYKY subunit (both with a 1.92 line). In agreement with previous experiments (Van Belzen and Albracht 1989), the amplitudes of the 1.92 and 1.86 lines changed in an identical way. It is therefore proposed that the cluster in the $75-\mathrm{kDa}$ subunit nearest to cluster $f$, i.e. cluster $e$, is responsible for the increase of the 1.86 line induced at $\mathrm{pH}$ 6.2. From the remaining four unassigned clusters, cluster $a$ cannot be reduced by NADH (Ohnishi 1998) and so the [2Fe-2S] cluster $c$ is responsible for the 1.94 line. This leaves the clusters $b$ and $d$ to account for contributions to the 1.88 line. Because the NADH-induced 1.88 line represents two spins per Complex I, it is proposed that the $\mathrm{pH}$-dependent increase of the 1.88 line in Fig. $4 \mathrm{a}$ mainly represents the cluster nearest to cluster $e$, i.e. cluster $d$, although a contribution of cluster $b$ cannot be excluded.

Thus, the $\mathrm{pH}$ jump from 8 to 6.2 resulted in the extra reduction by NADPH of three clusters. This event was followed by the re-oxidation of the clusters $f$ and $e$ (and part of the clusters contributing to the 1.88 line, i.e. the clusters $d$ and/or $b$; see block schemes in Fig. 4a). Because electrons started to disappear from Complex I after $280 \mathrm{~ms}$, it is assumed that electron transfer to the Q-pool became unlocked. At $800 \mathrm{~ms}$ and $\mathrm{pH} 6.2$ the clusters $g$ and $h$ remained reduced, whereas the clusters $f$ and $e$ were reoxidised. This implies that in active Complex I the ratelimiting step in the steady-state $\mathrm{NADPH} \rightarrow \mathrm{O}_{2}$ oxidation reaction at $\mathrm{pH} 6.2$ is the transfer of electrons from cluster $g$ to cluster $f$. At $800 \mathrm{~ms}, 40 \%$ of the 1.94 and 1.88 lines remained present. This shows that, for unknown reasons, electrons from the clusters $b, c$ and $d$ cannot reach the Qpool under the used conditions.

To verify the interpretation of Fig. $4 a$, the experiment was repeated with rotenone added to the syringe with the SMP (Fig. 4b). The traces show that the inhibitor did not affect the reaction of NADPH with SMP at $\mathrm{pH}$ 8.0, i.e. the degree of reduction at $1000 \mathrm{~ms}$ (zero time in the plot). Nor did rotenone affect the increase in amplitude of the lines after the $\mathrm{pH}$ jump (except for a slight effect on the 1.88 line in the 200 to $300 \mathrm{~ms}$ region). However, rotenone prevented the decrease of the lines, a decrease seen in Fig. 4a. It is concluded that the inhibitor blocks electron transfer from the clusters to the Q-pool.

In combination with Fig. 3a, the results in Fig. 4 show that at $\mathrm{pH} 8$ electron transfer from the clusters $h$ and $g$ (and FMN-a) to ubiquinone is nearly absent. They suggest that electron transfer from NADPH at $\mathrm{pH} 6.2$ proceeds via the sequence of the clusters $h \rightarrow g \rightarrow f \rightarrow e$. They also imply that for transfer of electrons to the $\mathrm{Q}$ pool, additional reduction of the clusters $f$ plus $e$ is required; the transfer to $\mathrm{Q}_{10}$ is blocked by rotenone. The apparent $\mathrm{pH}$-dependent switch in electron transfer from cluster $g$ to cluster $f$ in the TYKY subunits is indicated by a thin red arrow in Fig. 2.

Activation of Complex I in oxidized SMP by a pH jump from 8 to 6.2

To test the effects of $\mathrm{pH} 6.2$ on oxidized SMP, a threesyringe experiment was performed where the $\mathrm{pH}$ of the oxidized SMP was first altered from 8.0 to 6.2. After $100 \mathrm{~ms}$, an excess of NADPH was added. This is shown in Fig. 5. The level of appearance of the 1.92 and 1.86 lines at $280 \mathrm{~ms}$ was much less than that in the experiments of Fig. 4. After $400 \mathrm{~ms}$ the amplitudes of all lines was nearly the same as that observed in Fig. $4 \mathrm{a}$ at $800 \mathrm{~ms}$. This can be explained by assuming that during the $100 \mathrm{~ms}$ at 6.2 Complex I in oxidized SMP started to convert already from a de-activated state into an activated state. This activation proceeded after mixing with NADPH, finally resulting in both a rapid electron transfer from cluster $g$ to cluster $f$ and a (more rapid) one from clusters $f$ plus $e$ to $\mathrm{Q}_{10}$. The experiment is in agreement with the notion that the transfer of electrons from cluster $g$ to cluster $f$ is rate limiting in 


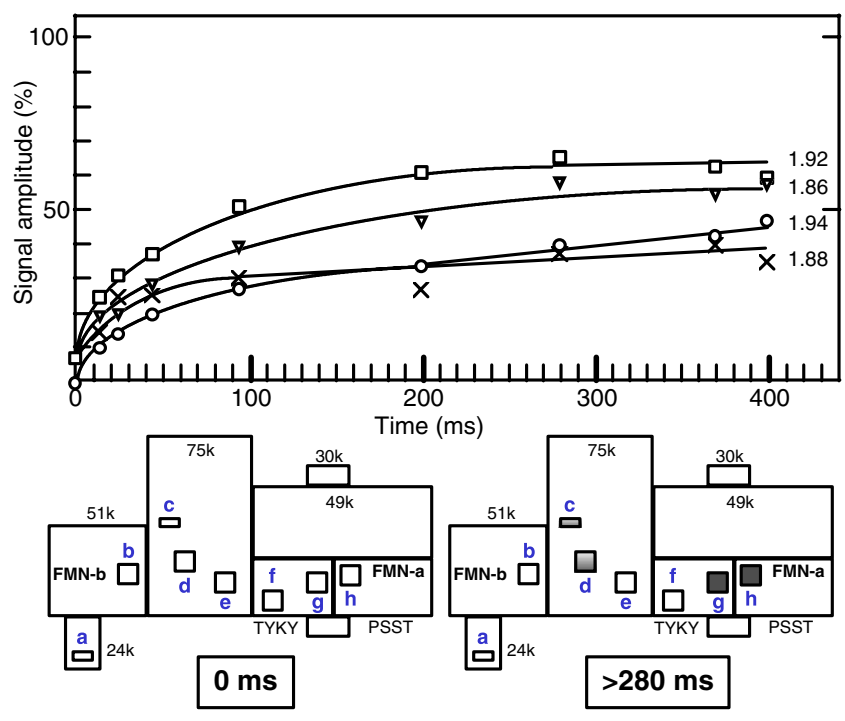

Fig. 5 Activation of Complex I in oxidized SMP by a $\mathrm{pH}$ jump from 8 to 6.2. a Three-syringe experiment in which syringe 1 contained SMP suspended in $0.25 \mathrm{M}$ sucrose, $1 \mathrm{mM}$ butanedione $(\mathrm{pH} 8.0$ ), syringe 2 held $0.25 \mathrm{M}$ sucrose, $300 \mathrm{mM}$ MES-KOH (pH 6.2) and syringe 3 was filled with $10 \mathrm{mM}$ NADPH, $0.25 \mathrm{M}$ sucrose $(\mathrm{pH} 8.0)$. The tubing connecting mixing chamber I with mixing chamber II was calibrated for a reaction time of $100 \mathrm{~ms}$. The moment that NADPH was added to the SMP in mixing chamber II was chosen as the zero time in the plot. The block schemes indicate the interpretation of the results. Left: redox states just before the addition of NADPH. Right: redox states at $280 \mathrm{~ms}$ and onwards. All symbols are the same as in Fig. 4

steady-state oxidation of NADPH by $\mathrm{O}_{2}$ catalyzed by SMP at $\mathrm{pH} 6.2$.

Striking differences between the $\mathrm{pH}$-dependent kinetic redox behaviour of the clusters $f$ plus $e$ and the clusters $b, c$ and $d$ upon reaction of SMP with NADPH

Figure 4 shows that the kinetics and the extent of appearance of the 1.94 (cluster $c$ ) and 1.88 (clusters $b$ plus $d$ ) lines induced by NADPH clearly differ from those of the 1.92 (clusters $f$ plus $g$ ) and 1.86 (clusters $e$ plus $h$ ) lines. Figure 6 shows an experiment where these differences are particularly clear. Submitochondrial particles in a rotenonecontaining medium ( $\mathrm{pH}$ 8.0) with a low buffering capacity were mixed with NADPH dissolved in a concentrated buffer that determined the final $\mathrm{pH}$ after mixing. The reaction, carried out at $\mathrm{pH}$ values between 5.5 and 8.3, was quenched after $100 \mathrm{~ms}$. Up to half (average of $40 \%$ ) of the lines at 1.92 and 1.86 developed within $100 \mathrm{~ms}$ in the $\mathrm{pH}$ region 7.75 to 8.3 . Full appearance of these lines occurred between $\mathrm{pH} 5.5$ and 7.2.

The amplitudes of the 1.92 and 1.86 lines showed a sharp change around $\mathrm{pH}$ 7.5. The behaviour of the 1.94 and 1.88 lines was completely different; both lines gradually increased with decreasing $\mathrm{pH}$. Hence, it is concluded that under the used conditions the clusters $b, c$ and $d$ are not in rapid redox equilibrium with any of the clusters $h, g, f$ or $e$. Inconsistent and unexplained behaviour of the 1.88 line, and in particular of the 1.94 line, has also been observed in previous experiments with NADPH (Bakker and Albracht 1986; Van Belzen and Albracht 1989).

Figure 6 also shows that in the $\mathrm{pH}$ range 5.5 to 7.2 the clusters $h, g, f$ and $e$ were, within error, completely reduced after $100 \mathrm{~ms}$. Thus, the transfer of electrons from cluster $g$ to cluster $f$ was unlocked under these conditions; rotenone prevented further transfer to ubiquinone.

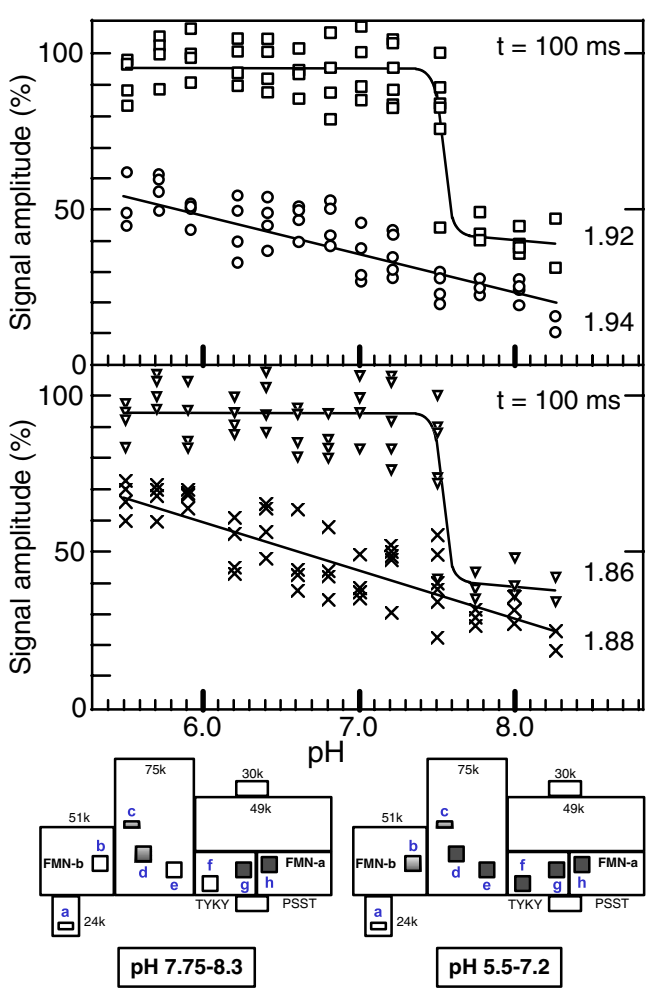

Fig. 6 Effects of NADPH and a simultaneous $p H$ jump on the appearance of EPR signals in Complex I as present in rotenoneinhibited SMP. This was a two-syringe experiment whereby after mixing, the SMP were exposed to two changes: an NADPH concentration of $5 \mathrm{mM}$ and a $\mathrm{pH}$ jump from $\mathrm{pH} 8.0$ to the indicated $\mathrm{pH}$ values. The reaction was quenched $100 \mathrm{~ms}$ after mixing. The SMP, suspended in $0.25 \mathrm{M}$ sucrose, $1 \mathrm{mM}$ butanedione and $45 \mu \mathrm{M}$ rotenone (pH 8.0; low buffering capacity) were mixed with $10 \mathrm{mM}$ NADPH dissolved in $0.25 \mathrm{M}$ sucrose, $1 \mathrm{mM}$ butanedione and various concentrated buffers: $200 \mathrm{mM}$ MES-KOH (pH 5.5 to 6.6 ), $200 \mathrm{mM}$ MOPS-KOH (pH 6.8 to 7.75) or $200 \mathrm{mM}$ Tris- $\mathrm{HCl}$ (pH 7.75 to 8.3). The maximal obtainable signal amplitudes were determined by replacing NADPH by NADH; in this case the levels of reduction were not $\mathrm{pH}$ dependent. The data points in the figure are the results of experiments with two separate batches of SMP (one of which was also used for the experiment in Fig. 3a) prepared from the same stock of mitochondria. With each batch, experiments at a certain $\mathrm{pH}$ value were carried out either in duplicate or in triplicate. All solutions were prepared in air and all handlings were in air as well. The block schemes indicate the interpretation of the results. Left: reduction with $\mathrm{NADPH}$ at $\mathrm{pH} 7.75$ to 8.3. Right: reduction with NADPH at $\mathrm{pH} 5.5$ to 7.2. All symbols are the same as in Fig. 4 
The effect of $N, N^{\prime}$-dicyclohexylcarbodi-imide (DCCD) on electron transfer in Complex I

DCCD, which readily reacts with carboxyl groups, inhibits the proton-translocation activities of several mitochondrial enzyme complexes. At concentrations two orders of magnitude higher than those required for inhibition of ATP synthase (Beechey et al. 1966; Beechey et al. 1967), it can inhibit the oxidation of NADH in uncoupled bovine SMP nearly completely without affecting the succinate oxidation (Beyer et al. 1972). From kinetic (hand mixing) EPR experiments and from measurements of the steadystate reduction of the Q pool in bovine-heart SMP ( $\mathrm{pH}$ 7.5), it was concluded that the site of inhibition of DCCD was between the Fe-S clusters and $\mathrm{Q}_{10}$ (Beyer et al. 1972; Krishnamoorthy and Hinkle 1988). Its effect on the $\mathrm{NADPH}$ oxidation has never been reported. This was studied in the following experiments.

Incubation of (non-coupled) SMP with $6 \mathrm{mM}$ DCCD led to an inhibition of $95 \%$ or more of the $\mathrm{NADH} \rightarrow \mathrm{O}_{2}$, $\mathrm{NADH} \rightarrow$ cytochrome $c$ and $\mathrm{NADH} \rightarrow \mathrm{Q}_{1}$ reactions (Fig. 7). The succinate $\rightarrow \mathrm{O}_{2}$ activity was inhibited by 12 to $13 \%$ and this was not dependent on the concentration of DCCD in the range of 0.1 to $6 \mathrm{mM}$. Hence, the used DCCD concentrations did not inhibit the Complexes III or IV. Pre-treatment of the SMP with butanedione did not influence the effects of DCCD. With NADH all Fe-S clusters in Complex I were fully reduced within $6 \mathrm{~ms}$, even at $4^{\circ} \mathrm{C}$ (not shown). This confirms and extends earlier experiments involving reaction times which were longer by more than three orders of magnitude (Beyer et al. 1972; Krishnamoorthy and Hinkle 1988). Thus, at a $6 \mathrm{~ms}$ time scale, DCCD does not interfere with NADH-induced electron transfer between the Fe-S clusters in Complex I and this is in agreement with the notion that DCCD inhibits transfer of electrons between the $\mathrm{Fe}-\mathrm{S}$ clusters and ubiquinone.

The effects of DCCD on the reactions of NADPH at $\mathrm{pH} 8$ and 6.2 with SMP pre-treated with butanedione are shown in Fig. 8. At $\mathrm{pH} 8.0$, when there is hardly any electron transfer from NADPH to $\mathrm{O}_{2}$ even in the absence of DCCD, full reduction of the clusters $h$ and $g$ and partial reduction of clusters $c$ and $d$ was observed, consistent with the results up to now. At pH 6.2, however, the clusters $d$ to $h$ were nearly fully reduced within $30 \mathrm{~ms}$ (and cluster $c$ was reduced to $30 \%$ ). In particular the appearance of the amplitudes of the 1.92 and 1.86 lines is directly comparable to similar experiments at $\mathrm{pH} 6.2$ in which rotenone was present, or where $\mathrm{Q}_{10}$-depleted SMP were used (Van Belzen and Albracht 1989). It is concluded that reoxidation of the clusters $f$ and $e$ by the Q-pool is inhibited by DCCD.

It has been reported that DCCD binds mainly to the ND1 subunit in Hatefi's Complex I (Yagi 1987; Yagi and Hatefi
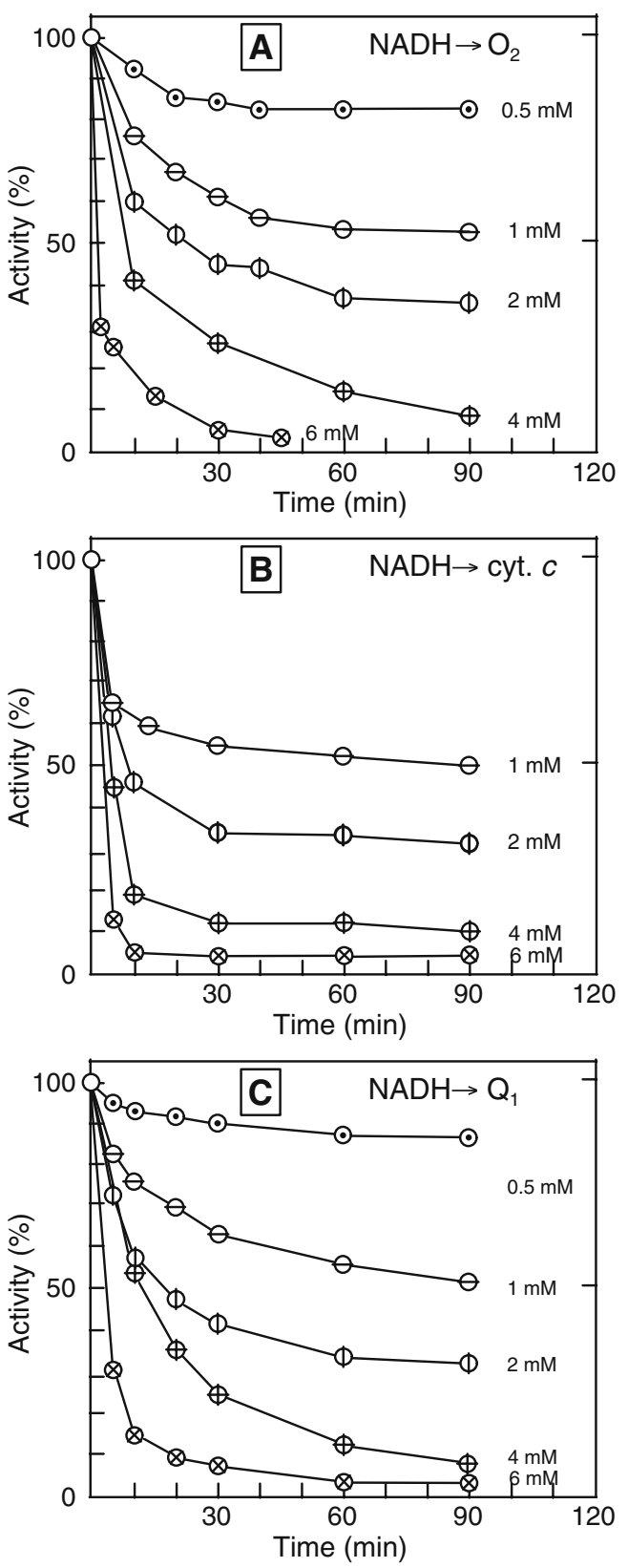

Fig. 7 The inhibition of several NADH-oxidation activities catalyzed by SMP (not treated with butanedione) by the incubation with DCCD at $\mathrm{pH} 8.0$ and $30^{\circ} \mathrm{C}$. A Effect on the $\mathrm{NADH} \rightarrow \mathrm{O}_{2}$ reaction (maximal activity $1235 \mathrm{nmol}$ of NADH per min per mg of protein). B Effect on the NADH $\rightarrow$ cytochrome $c$ reaction (maximal activity $920 \mathrm{nmol}$ of cytochrome $c$ per min per mg protein). (C) Effect on the $\mathrm{NADH} \rightarrow \mathrm{Q}_{1}$ reaction (maximal activity $735 \mathrm{nmol}$ of NADH per min per $\mathrm{mg}$ protein). The assays were performed as described in the section Materials and methods. Activity of untreated SMP (open circles) or treated with $0.5 \mathrm{mM}$ DCCD (circles with dot), $1 \mathrm{mM}$ DCCD (circles with horizontal bar), $2 \mathrm{mM}$ DCCD (circles with vertical bar), $4 \mathrm{mM}$ DCCD (circles with + sign) or 6 mM DCCD (circles with $\mathrm{x}$ sign)

1988). The labelling was not affected by the complete inhibition by rotenone, although the ND1 subunit can bind this inhibitor as well (Earley and Ragan 1984; Earley et al. 1987). Also piericidin A can bind to ND1 (Murai et al. 
Fig. 8 Reduction by NADPH of Complex I in SMP pre-treated with butanedione, and subsequently with $6 \mathrm{mM} \mathrm{DCCD}$ for $60 \mathrm{~min}$. This was a two-syringe experiment. A Kinetics in $0.25 \mathrm{M}$ sucrose, $100 \mathrm{mM}$ Tris$\mathrm{HCl}, 1 \mathrm{mM}$ butanedione $(\mathrm{pH}$ 8.0). B Kinetics in $0.25 \mathrm{M}$ sucrose, $100 \mathrm{mM}$ MES-KOH buffer and $1 \mathrm{mM}$ butanedione (pH 6.2). The NADPH concentration in syringe 2 was $10 \mathrm{mM}$. The block schemes indicate the interpretation of the results. Left: redox states at $50 \mathrm{~ms}$ and onwards after mixing with $\mathrm{NADPH}$ at $\mathrm{pH}$ 8.0. Right: redox states at $30 \mathrm{~ms}$ and onwards after mixing with $\mathrm{NADPH}$ at $\mathrm{pH}$ 6.2. All symbols are the same as in Fig. 4
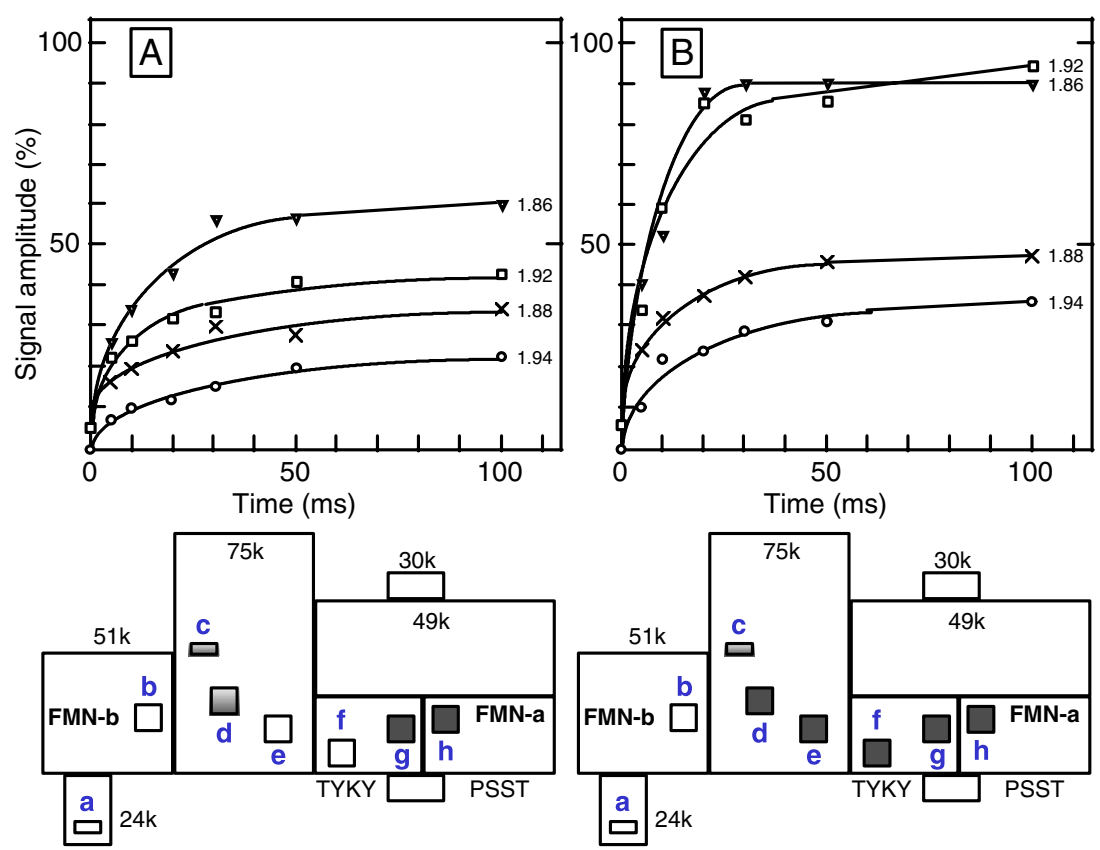

$>50 \mathrm{~ms}(\mathrm{pH} 8.0)$
$>30 \mathrm{~ms}(\mathrm{pH} \mathrm{6.2)}$
2007). Figure 9 shows that the binding of DCCD had no effect on the binding of piericidin A. The number of piericidin A molecules required for maximal inhibition of the $\mathrm{NADH} \rightarrow \mathrm{O}_{2}$ activity was equal to the number of spins represented by the 1.92 line (clusters $f$ plus $g$ ), i.e. two per Complex I. This is in accordance with binding studies of

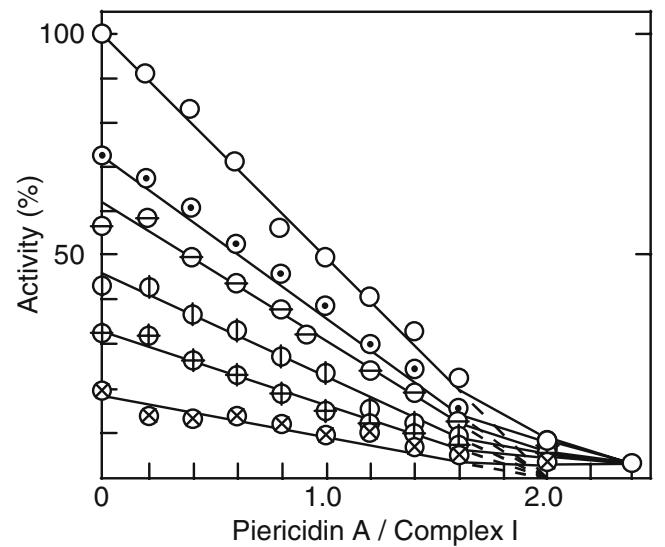

Fig. 9 Titration with piericidin $A$ of the steady-state $\mathrm{NADH} \rightarrow \mathrm{O}_{2}$ reaction catalyzed by SMP treated with different concentrations of $D C C D$. The activities are shown relative to that of untreated SMP (1065 nmol of NADH per min per mg of protein at $\mathrm{pH} 8.0$ and $30^{\circ} \mathrm{C}$ ). Open circles, not treated by DCCD; circles with dot, treated with $0.125 \mathrm{mM}$ DCCD for $30 \mathrm{~min}$; circles with horizontal bar, treated with $1 \mathrm{mM}$ DCCD for $30 \mathrm{~min}$; circles with vertical bar, treated with $2 \mathrm{mM}$ DCCD for $30 \mathrm{~min}$; circles with + sign, treated with $4 \mathrm{mM} \mathrm{DCCD} \mathrm{for}$ $30 \mathrm{~min}$; circles with $\mathrm{x}$ sign, treated with $4 \mathrm{mM}$ DCCD for $60 \mathrm{~min}$. The absolute spin concentration represented by the N2 EPR signal from the SMP was determined (Albracht et al. 1979; Van Belzen et al. 1990) and the Complex I concentration was taken as half that value the ${ }^{14}$ C-labelled inhibitor to SMP (Gutman et al. 1970). When $35 \mu \mathrm{M}$ NADPH was present during the preincubation with piericidin A, the DCCD-treated SMP required only one molecule of piericidin A per Complex I to fully inhibit the initial $\mathrm{NADH} \rightarrow \mathrm{O}_{2}$ reaction, although the steady-state reaction was $50 \%$ inhibited (not shown). These results are the same as earlier found for the inhibition by piericidin A of the NADH oxidation catalyzed by SMP in the absence of DCCD (Van Belzen et al. 1990).

\section{Discussion}

The conclusions from the freeze-quench experiments led to the working hypothesis presented in Fig. 2. It is proposed that NADH and NADPH, which have standard redox potentials differing by only $5 \mathrm{mV}$ (Hatefi and Yamaguchi 1996), react at opposite ends of the chain of redox groups and that $\mathrm{Q}_{10}$ reacts in between the two dehydrogenase modules close to cluster $f$. The model is consistent with optical experiments at neutral $\mathrm{pH}$ from the time that the cubane clusters were not yet discovered. NADH causes a maximal bleaching of the yellow colour (450 to $475 \mathrm{~nm}$ region) of Hatefi's Complex I under aerobic conditions (Hatefi 1968). In the presence of rotenone the bleaching is ca. 50\% (Hatefi 1968), although, as measured later on (Beinert and Albracht 1982), the reduction of the Fe-S clusters is not affected. Subsequent addition of NADPH leads to $100 \%$ bleaching. The additional bleaching is thus caused by reduction of a yellow non-Fe chromophore. In 
turn, NADPH alone induces a 50\% bleaching not affected by rotenone; subsequent addition of NADH extends the bleaching to $100 \%$. Such observations were also made with SMP (Hatefi and Hanstein 1973). It is proposed here that the NADPH-induced bleaching is due to FMN-a in the PSST subunit.

Ragan measured the bleaching at $450 \mathrm{~nm}$ of Hatefi's Complex I with NADPH in air (50\% bleaching) and under near anaerobic conditions ( $91 \%$ bleaching). Various levels of bleaching could also be obtained with an adjustable NADH-generating system. Hence, it was concluded that the $50 \%$ bleaching by NADPH can be explained by "autoxidation of an enzyme component at a higher rate than its reduction by NADPH" (Ragan 1976). It is proposed here that this component is FMN-b. Thus, in addition to its rapid reaction site at FMN-a, NADPH can also input electrons at FMN-b at a rate slower than the rate of auto-oxidation of reduced FMN-b by $\mathrm{O}_{2}$. This is in line with the finding that in Singer's NADH dehydrogenase the N1b EPR signal (from cluster $c$ ) can be completely induced with NADPH under anaerobic conditions if one waits long enough (halftime $20 \mathrm{~s}$ at $22^{\circ} \mathrm{C}$ ) (Beinert et al. 1965). Apparently, this extremely slow reduction proceeds via FMN-b.

Pyridine-nucleotide binding sites in Complex I

Both the 51-kDa subunit (Pilkington et al. 1991) and 39$\mathrm{kDa}$ subunit (Fearnley and Walker 1992) from the bovine enzyme contain a $\beta$-sheet: $\alpha$-helix: $\beta$-sheet nucleotide binding fold. The motif in the $51-\mathrm{kDa}$ subunit is conserved in homologous subunits of $N$. crassa, Y. lipolytica, $P$. denitrificans and E. coli and the homologous HoxF subunit of the soluble $\mathrm{NAD}^{+}$-reducing [NiFe]-hydrogenase from Ralstonia eutropha. This is the NADH-binding site. In the T. thermophilus enzyme the nicotinamide ring of the bound NADH is positioned on top of the isoalloxazine ring of FMN, like in many other pyridinenucleotide-binding flavoenzymes (Berrisford and Sazanov 2009). Also the motif in the $39-\mathrm{kDa}$ subunit is conserved in homologous subunits of the non-mammalian mitochondrial enzymes. The (hydrophobic) 39-kDa subunit from the bovine enzyme can bind to an $\mathrm{NADP}^{+}$-agarose column and can be eluted with NADPH (Yamaguchi et al. 1998). This subunit did not bind NAD $(\mathrm{H})$. The NADH dehydrogenases from $N$. crassa and Y. lipolytica contain a tightlybound NADPH molecule; mutation studies indicated that also in this case the presumable binding site is the $39-\mathrm{kDa}$ subunit (Schulte et al. 1999; Abdrakhmanova et al. 2006). Although the $N$. crassa enzyme oxidizes NADPH in air at a very low rate, the bound NADPH is not oxidized (Schulte et al. 1999). An homologous subunit is apparently missing in some prokaryotic enzymes ((Schulte et al. 1999) and a search in the UniProtKB database). It has not been established whether the $39-\mathrm{kDa}$ subunit is essential for the reaction of Complex I with NADPH.

Photoaffinity labelling studies with NAD $(\mathrm{H}), \operatorname{NADP}(\mathrm{H})$ and ADP showed that five polypeptides in Hatefi's Complex I can be marked. They have apparent molecular masses of $51,42,39,30$ and 18 to $20 \mathrm{kDa}$ (gel electrophoresis) (Yamaguchi et al. 2000). The 51 and 30$\mathrm{kDa}$ polypeptides became labelled with all three nucleotides, while the $42-\mathrm{kDa}$ one mainly reacted with $\mathrm{NAD}(\mathrm{H})$, but not NADP(H). The 39-kDa polypeptide did not react with NADP(H) unless $2 \%$ Trition X-100 was added to Complex I; it did not react with $\operatorname{NAD}(\mathrm{H})$. The 18 to $20-\mathrm{kDa}$ polypeptide was the only one that reacted exclusively with NADPH. In earlier publications (Belogrudov and Hatefi 1994; Belogrudov and Hatefi 1996) the investigators mentioned that a $20-\mathrm{kDa}$ subunit was one of the 31 subunits of the hydrophobic-protein (HP) domain that can be obtained by resolution of Complex I with chaotropic agents (Hatefi and Stempel 1967) and that this subunit contained a motif of three Cys residues that could possibly bind a tetranuclear Fe-S cluster. Obviously, this subunit is the PSST subunit. The iron-sulfur protein (IP) domain of Complex I also contains a $18-\mathrm{kDa}$ subunit (Belogrudov and Hatefi 1994) with AQDQ as the first four residues (Fearnley and Walker 1992). This subunit is proposed to modulate the activity of Complex I by way of proteinekinase-A-mediated phosphorylation of a Ser residue by cAMP in its C-terminus (Papa et al. 2008). Further subunits with a similar molecular mass (gel electrophoresis) are B22 (22 kDa), PDSW (22 kDa), ASHI (19 kDa) and B18 (18 kDa) (Walker et al. 1992). Hence, the identity of 'the 18-20-kDa polypeptide' of Hatefi's Complex I that was exclusively labelled with NADPH (Yamaguchi et al. 2000) remains unclear.

The evolutionary-related soluble $\mathrm{NAD}^{+}$-reducing [NiFe]-hydrogenase from $R$. eutropha requires the presence of two Hox I (18.8 kDa) subunits, as well as a second flavin similar to FMN-a (Van der Linden et al. 2004), for its specific reaction with NADPH. It has been speculated that two adjacent HoxI subunits, each having a mononucleotidebinding site, can build a dinucleotide-binding domain for the binding of NADPH (Burgdorf et al. 2005).

Kinetic issues. Reactions with $\mathrm{NAD}(\mathrm{P}) \mathrm{H}$

In the bovine enzyme full reduction of the clusters $b$ to $h$ by NADH was obtained within $6 \mathrm{~ms}$ at all $\mathrm{pH}$ values used for Fig. 3a. Assuming the reduction of the FMN-b group (the rate of reduction of FMN-a is not known) and seven reducible Fe-S clusters per Complex I, this means that at least five molecules of NADH are oxidized by Complex I within this time, leading to a turnover number for NADH of at least $830 \mathrm{~s}^{-1}$ (at $22^{\circ} \mathrm{C}$ ). The real turnover number must 
be greater than $3300 \mathrm{~s}^{-1}$, because full reduction is also obtained within $6 \mathrm{~ms}$ at $4^{\circ} \mathrm{C}$.

The spin concentration of the N2 signal in bovine SMP, as routinely determined by EPR, amounts to a content of $0.12 \mathrm{nmol}$ per $\mathrm{mg}$ protein (Van Belzen et al. 1990); this means that the Complex I content is $0.06 \mathrm{nmol} . \mathrm{mg}^{-1}$. An estimate of this content can also be obtained from the ratio of the concentrations of the Complexes I and III, determined by EPR in ten batches of SMP, which is 0.14 to 1 , respectively (Albracht et al. 1980; Beinert and Albracht 1982). Assuming a cytochrome $c_{1}$ content in bovine SMP of $0.46 \mathrm{nmol}$ per $\mathrm{mg}$ of protein (Vanneste 1966; Orme-Johnson et al. 1974b), the Complex I content amounts to $0.064 \mathrm{nmol} . \mathrm{mg}^{-1}$.

The observed maximal $\mathrm{NADH} \rightarrow \mathrm{O}_{2}$ rate is $1100 \mathrm{nmol}$. $\mathrm{min}^{-1} . \mathrm{mg}^{-1}$ at $30^{\circ} \mathrm{C}$ (Fig. 3). With $0.06 \mathrm{nmol}$ Complex I per $\mathrm{mg}$ of protein, this is equivalent to a turnover number for NADH of $306 \mathrm{~s}^{-1}$ (and ca. $153 \mathrm{~s}^{-1}$ or less at $22^{\circ} \mathrm{C}$; see Table 2). During this reaction all clusters remain fully reduced (De Jong et al. 1994). These data are in line with the well known property of the enzyme that its rate of reduction by NADH is much faster than its rate of reoxidation by ubiquinone. Freeze-quench experiments with NADH do not disclose at what point in the enzyme electrons leave to the Q-pool.

The $\mathrm{NADPH} \rightarrow \mathrm{O}_{2}$ rate at $30^{\circ} \mathrm{C}$ and $\mathrm{pH} 8$ is ca. $10 \mathrm{nmol}$. $\mathrm{min}^{-1} \cdot \mathrm{mg}^{-1}$ (Fig. 3a), equivalent to a turnover number for NADPH of $2.8 \mathrm{~s}^{-1}$ (i.e. ca. $1.4 \mathrm{~s}^{-1}$ or less at $22^{\circ} \mathrm{C}$ ). Yet the clusters $h$ (PSST subunit) and $g$ (TYKY subunit), and also FMN-a, are reduced in ca. $50 \mathrm{~ms}$, giving a turnover number for NADPH of $40 \mathrm{~s}^{-1}$ (at $22^{\circ} \mathrm{C}$ ). It is likely that part of the $\mathrm{O}_{2}$ consumption is caused by the very slow reduction by NADPH of FMN-b and the faster auto-oxidation of this reduced flavin by $\mathrm{O}_{2}$. In addition a contribution of a possible auto-oxidation of reduced FMN-a cannot be excluded. The data are in agreement with the conclusion that at $\mathrm{pH} 8 \mathrm{FMN}-\mathrm{a}$ and the clusters $h$ and $g$ do not react with ubiquinone and that the transfer of electrons from cluster $g$ to cluster $f$ in the TYKY subunit is not functioning at this $\mathrm{pH}$, although the reverse reaction is. Inhibitors like rotenone (Fig. 4b) or DCCD (Fig. 8a) do not affect this behaviour. Transfer of electrons from cluster $g$ to cluster $f$ is unlocked by decreasing the $\mathrm{pH}$ from 8 to 6.2. With NADPH-reduced SMP this unlocking process takes at least $280 \mathrm{~ms}$ (Fig. 4). It is unlikely that the effect of $\mathrm{pH}$ on the reduction of cluster $f$ by NADPH is due to a $\mathrm{pH}$-dependent redox potential of this cluster. In that case a similar effect should occur with NADH as reductant.

A second $\mathrm{pH}$-dependent switch must be activated to allow oxidation of the NADPH-reduced clusters $f$ and $e$ by the Qpool at pH 6.2 (Fig. 4). Once unlocked, this oxidation is inhibited by rotenone or DCCD. The inhibition by DCCD is consistent with a direct coupling between electron transfer and proton translocation as earlier suggested for the oxidation of NADH (Yagi 1987; Yagi and Hatefi 1988). Rotenone and piericidin A can each bind to two sites, one close to a site in the $49-\mathrm{kDa}$ subunit, the site structurally equivalent to that of the $\mathrm{Ni}-\mathrm{Fe}$ site in $[\mathrm{NiFe}]-$ hydrogenases (Darrouzet et al. 1998; Prieur et al. 2001), and the other on

Table 2 Kinetics of the reactions of NADH and NADPH with bovine Complex I-The $\mathrm{NAD}(\mathrm{P}) \mathrm{H} \rightarrow \mathrm{FMN}$ data are from freeze-quench

are from steady-state experiments. All turnover numbers refer to $\mathrm{NAD}(\mathrm{P}) \mathrm{H}$ consumption and are standardised to $22^{\circ} \mathrm{C}$ assuming a twofold change in rate per $10^{\circ} \mathrm{C}$

\begin{tabular}{lllll}
\hline Reaction & $\mathrm{pH}$ & $\mathrm{TON}\left(\mathrm{s}^{-1}, 22^{\circ} \mathrm{C}\right)$ & Preparation & Reference \\
\hline $\mathrm{NADH} \rightarrow \mathrm{FMN}-\mathrm{b}$ & $6.5-8.5$ & $>3300$ & SMP & This study \\
$\mathrm{NADH} \rightarrow \mathrm{O}_{2}$ & 7.5 & $<153$ & SMP & This study \\
$\mathrm{NADPH} \rightarrow \mathrm{FMN}-\mathrm{a}$ & 8 & 40 & SMP & This study \\
$\mathrm{NADPH} \rightarrow \mathrm{O}_{2}$ & 8 & $<1.4$ & SMP & This study \\
$\mathrm{NADPH} \rightarrow \mathrm{FMN}-\mathrm{a}$ & 6.2 & 100 & SMP & This study \\
$\mathrm{NADPH} \rightarrow \mathrm{O}_{2}$ & 6.2 & $<34$ & SMP & This study \\
$\mathrm{NADH} \rightarrow \mathrm{K}_{3} \mathrm{Fe}(\mathrm{CN})_{6}{ }^{\mathrm{a}}$ & 7.5 & $\sim 2800$ & Hatefi's C-I & (Hatefi and Stempel 1969) \\
$\mathrm{NADH} \rightarrow \mathrm{K}_{3} \mathrm{Fe}(\mathrm{CN})_{6}{ }^{\mathrm{b}}$ & 7.5 & $\sim 2800$ & Hatefi's C-I & (Ragan et al. 1974) \\
$\mathrm{NADH} \rightarrow \mathrm{K}_{3} \mathrm{Fe}(\mathrm{CN})_{6}{ }^{\mathrm{c}}$ & 7.55 & $\sim 8000$ & Hatefi's C-I & (Dooijewaard and Slater 1976) \\
$\mathrm{NADPH} \rightarrow \mathrm{K}_{3} \mathrm{Fe}(\mathrm{CN})_{6}$ & 7.5 & n.detect. & Hatefi's C-I & (Hatefi et al. 1962) \\
$\mathrm{NADPH} \rightarrow \mathrm{K}_{3} \mathrm{Fe}(\mathrm{CN})_{6}$ & 7.4 & n.detect. & Singer's dh & (Ringler et al. 1960) \\
$\mathrm{NADPH} \rightarrow \mathrm{K}_{3} \mathrm{Fe}(\mathrm{CN})_{6}{ }^{\mathrm{d}}$ & 6.5 & $<7$ & Hatefi's C-I & (Hatefi and Hanstein 1973) \\
$\mathrm{NADPH} \rightarrow \mathrm{K}_{3} \mathrm{Fe}(\mathrm{CN})_{6}{ }^{\mathrm{e}}$ & 5.5 & $<12$ & Hatefi's C-I & (Ragan et al. 1974) \\
\hline
\end{tabular}

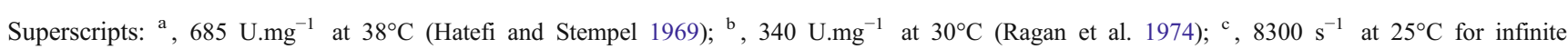
concentration of both substrates and disregarding the double substrate inhibition. This rate represents the dissociation of $\mathrm{NAD}^{+}$bound to the reduced enzyme (Dooijewaard and Slater 1976); ${ }^{\mathrm{d}}, 1.7 \mathrm{U} \cdot \mathrm{mg}^{-1}$ at $38^{\circ} \mathrm{C}$ (Hatefi and Hanstein 1973); ${ }^{\mathrm{e}}, 3.04 \mathrm{U} \cdot \mathrm{mg}^{-1}$ at $38^{\circ} \mathrm{C}$ (Ragan et al. 1974); ${ }^{\mathrm{f}}$, In the presence of rotenone, DCCD or in $\mathrm{Q}_{10}$-depleted SMP. Abbreviations: TON, turnover number for NAD(P)H; Hatefi's C-I, Hatefi's Complex I; Singer's dh, Singer's NADH dehydrogenase; n.detect., not detectable; $\mathrm{U}, \mu \mathrm{mol} \cdot \mathrm{min}^{-1}$ 
the ND1 subunit (Earley and Ragan 1984; Earley et al. 1987; Murai et al. 2007). It is hypothesised here that also the inhibition by these poisons is primarily due to the blockage of proton transfer, resulting in inhibition of electron transfer.

In Fig. 8b, an experiment at $\mathrm{pH} 6.2$ (with active Complex I) where electron transfer to the Q-pool is blocked by DCCD, the 1.92 and 1.86 lines are fully developed by $\mathrm{NADPH}$ in $30 \mathrm{~ms}$ at $\mathrm{pH} 6.2$ and $22^{\circ} \mathrm{C}$. Reduction of four clusters and one flavin per Complex I within $30 \mathrm{~ms}$ requires three molecules of NADPH, resulting in a turnover number for NADPH of $100 \mathrm{~s}^{-1}$ (Table 2). The $\mathrm{NADPH} \rightarrow \mathrm{O}_{2}$ reaction at $\mathrm{pH} 6.2$ (and $30^{\circ} \mathrm{C}$ ) is $250 \mathrm{nmol} \cdot \mathrm{min}^{-1} \cdot \mathrm{mg}^{-1}$ (Fig. 3a), equivalent to a turnover number for NADPH of $69 \mathrm{~s}^{-1}$ (i.e. ca. $34 \mathrm{~s}^{-1}$ or less at $22^{\circ} \mathrm{C}$ ). The numbers show that the rate of reaction of active Complex I in SMP with NADPH at $\mathrm{pH} 6.2$ in the presence of DCCD (or with rotenone, or in $\mathrm{Q}_{10}$-depleted SMP (Van Belzen and Albracht 1989)) is faster than the overall NADPH oxidation rate in the absence of inhibitors. During the latter reaction the clusters $f$ and $e$ remain oxidized. This implies that electron transfer from cluster $g$ to cluster $f$ becomes rate limiting ( $<68$ electrons per second) and slower than the rate in the presence of inhibitors $\left(200 \mathrm{~s}^{-1}\right)$. In other words, when the complete enzyme starts to function, i.e. electron transfer from NADPH to $\mathrm{Q}_{10}$ with concomitant proton translocation, the rate of electron transfer from cluster $g$ to cluster $f$ seems to slow down. Figure 3 a shows that the rates of the $\mathrm{NADPH} \rightarrow \mathrm{O}_{2}$ reaction at $\mathrm{pH} 6.2$ and 6.4 are ten-fold higher than those at $\mathrm{pH} 7.2$ and 7.4, respectively. Thus, the rate is apparently proportional to the proton concentration and hence the steady-state electron transfer from cluster $g$ to cluster $f$ may be limited by the supply of protons from the medium. Whether this is part of the proton-translocation machinery remains to be investigated.

\section{Kinetic issues. The $\mathrm{NAD}(\mathrm{P}) \mathrm{H} \rightarrow \mathrm{K}_{3} \mathrm{Fe}(\mathrm{CN})_{6}$ reaction}

The turnover number for NADPH reacting with Complex I in SMP at $\mathrm{pH} 8$ determined in this study is $40 \mathrm{~s}^{-1}$ at $22^{\circ} \mathrm{C}$ (Table 2). If the released electrons would enter the complex via FMN-b, it would be expected that $\mathrm{K}_{3} \mathrm{Fe}(\mathrm{CN})_{6}$ could remove them again at a high rate, just as is the case with $\mathrm{NADH}$ as electron donor (Table 2). For the purified complex $(980 \mathrm{kDa})$ this would result in an $\mathrm{NADPH} \rightarrow \mathrm{K}_{3} \mathrm{Fe}(\mathrm{CN})_{6}$ activity of $2.4 \mu \mathrm{mol} \cdot \mathrm{min}^{-1} \cdot \mathrm{mg}^{-1}$, which would be easily detectable in a standard assay. For Hatefi's Complex I and Singer's NADH-dehydrogenase no $\mathrm{NADPH} \rightarrow \mathrm{K}_{3} \mathrm{Fe}(\mathrm{CN})_{6}$ activity could be detected at neutral $\mathrm{pH}$ (Table 2). Some activity could only be observed with high enzyme concentrations and at lower $\mathrm{pH}$ values at $38^{\circ} \mathrm{C}$ (Table 2). Even then the rate (ca. $10 \mathrm{~s}^{-1}$ ) is ten-fold lower than that of the reaction of NADPH with Complex I in SMP determined in the present study $\left(100 \mathrm{~s}^{-1}\right)$. Thus, the absence of the $\mathrm{NADPH} \rightarrow \mathrm{K}_{3} \mathrm{Fe}(\mathrm{CN})_{6}$ activity of the classical bovine enzyme preparations is consistent with the conclusion that the rapid reduction of the clusters $h$ and $f$ by NADPH in SMP does not involve FMN-b.

Kinetic issues. The reaction with $\mathrm{Q}_{10}$

Reduction of Complex I by NADH is at least 20-fold faster than its reoxidation by $\mathrm{Q}_{10}$ (Table 2). In SMP with active Complex I a ubisemiquinone radical is evoked ca. $40 \mathrm{~ms}$ after mixing with NADH at pH 7.5 (De Jong and Albracht 1994). The signal amounts to one spin per Complex I (De Jong et al. 1994), remains present during steady-state electron transfer, but disappears when electron transfer comes to a halt. In tightly-coupled SMP the spin-relaxation rate of this radical is enhanced, in an uncoupler-sensitive way, by spin-spin interaction with one (or two) Fe-S cluster (s) showing a $g_{1}=2.05$ line. In view of the fact that both the clusters $f$ and $g$ have such a 2.05 line, the precise interpretation of the observed magnetic interactions (Van Belzen et al. 1997; Yano et al. 2005) deserves further attention. It is proposed here that at least one of the clusters in the TYKY subunit, most likely cluster $f$, is in contact with this ubisemiquinone. Subsequent transfer of electrons proceeds presumably via reaction of the bound ubisemiquinone with quinone molecules from the Q-pool. It has been suggested that the wide hydrophobic cleft formed by the 49-kDa and PSST subunits (Fig. 2) plays an important role in the reaction with $\mathrm{Q}_{10}$ (Sazanov and Hinchliffe 2006; Fontecilla-Camps et al. 2007). In this connection it is worthwhile to recall that there is as yet no rational explanation for the finding that reconstitution of the $\mathrm{NADH} \rightarrow \mathrm{O}_{2}$ activity of Q-depleted bovine SMP is specific for ubiquinones with a certain length of the isoprenoid side chain (7 to 10 units), whereas restoration of the succinate $\rightarrow \mathrm{O}_{2}$ activity displays no such specificity (Lenaz et al. 1968; Lenaz et al. 1971; Lenaz 1998). A reaction of $Q_{10}$, which has a length of ca. $50 \AA$ in its stretched conformation, deep inside the hydrophobic cleft and dependent on the presence of phospholipids would be in agreement with these observations.

In NADPH-reduced Complex I not all Fe-S clusters are in rapid redox equilibrium

This is especially apparent in Fig. 6. At pH 7.6-8.3 the clusters $g$ and $h$ are not in redox equilibrium with any of the other clusters. At pH 5.5-7.4 the clusters $b, c$ and $d$ are not in rapid redox equilibrium with the clusters $e, f, g$ or $h$. It is proposed here that the reduction of the clusters $b, c$ and $d$ is caused by electrons that escaped from the very slow steadystate $\mathrm{NADPH} \rightarrow \mathrm{O}_{2}$ reaction catalyzed by (auto-oxidation of) FMN-b. It is not understood why electrons in these clusters 
do not flow to the Q-pool under conditions, where electrons coming in via FMN-a do. It has earlier been observed that when aerobic SMP (with an inactivated transhydrogenase) were mixed with $25 \mu \mathrm{M}$ NADH plus $5 \mathrm{mM} \mathrm{NADPH}$, all Fe$\mathrm{S}$ EPR signals were fully developed within $5 \mathrm{~ms}$. After $400 \mathrm{~ms}$, when the NADH was used up, the 1.94 line (cluster c) disappeared, but the 1.92, 1.86 and 1.88 lines remained present at $50 \%, 40 \%$ and $25 \%$, respectively, for at least $30 \mathrm{~s}$ (Fig. 2 in (Albracht and Bakker 1986)).

The redox potential of NADPH increases with decreasing $\mathrm{pH}$. Yet, the level of reduction of the clusters $b, c$ and $d$ in Fig. 6 increases linearly. This can be explained by an increase of the very slow NADPH $\rightarrow$ FMN-b reaction rate due to neutralisation of the negative charge of the $2^{\prime}$ phosphate group of NADPH.

Although the sharp decrease in the reduction levels of the clusters $f$ and $e$ above $\mathrm{pH} 7.5$ (Fig. 6) may point to a possible effect of the Tris buffer, this possibility can be eliminated. The reaction of SMP with NADPH at $\mathrm{pH} 8$ for $1000 \mathrm{~ms}$ in the absence of added buffer (zero points in Fig. 4a and b) resulted in the same redox changes as those in the range of pH 7.75 to 8.3 in the presence of Tris buffer (Fig. 6). In addition, no such sudden changes with $\mathrm{pH}$ were observed in the steady-state activity measurements (Fig. 3a), although the same sets of buffers were used. Apparently, the de-active (above $\mathrm{pH}$ 7.6) and active (below $\mathrm{pH}$ 7.4) conformations of the enzyme are not in a pH-dependent equilibrium. In the experiment the conversion of de-active to active enzyme is apparently triggered by a decrease of the $\mathrm{pH}$ from 8 to 7.4 or lower. At pH 8 activation can be induced by turnover of a small amount of NADH (Kotlyar and Vinogradov 1990) or by incubation with $35 \mu \mathrm{M}$ of NADPH for $5 \mathrm{~min}$ (Van Belzen et al. 1990). The active state (at pH 8) is stable for hours at $0^{\circ} \mathrm{C}$ but converts back to the de-active state within ca. $5 \mathrm{~min}$ at $33^{\circ} \mathrm{C}$ (Kotlyar and Vinogradov 1990).

There is little doubt that at neutral $\mathrm{pH}$ the reaction of Complex I with NADPH is hardly contributing to the overall electron transfer to the Q-pool. It is anticipated that NADPH in the mitochondrial matrix can keep FMN-a and the clusters $h$ and $g$ permanently reduced. The degree of reduction will depend on the NADPH/NADP ${ }^{+}$ratio. These reducing equivalents in the enzyme may play a role in the regulation of ROS (radical oxygen species) production by Complex I, dependent on the NADPH/NADP ${ }^{+}$ratio (Albracht, S.P.J., Meijer, A.J. and Rydström, J., manuscript in preparation).

\footnotetext{
Acknowledgements At the time the work was supported by grants from The Netherlands Organization for the Advancement of pure Research (N.W.O.) under the auspices of the Netherlands Foundation for Chemical Research (S.O.N.). SPJA wishes to express his thanks to A.J. (Fred) Meijer (Amsterdam), Jan Rydström (Göteborg), Simon De Vries (Delft), and especially to E.C. (Bill) Slater (Painswick), for their critical and constructive comments on a preliminary form of this paper.
}

Open Access This article is distributed under the terms of the Creative Commons Attribution Noncommercial License which permits any noncommercial use, distribution, and reproduction in any medium, provided the original author(s) and source are credited.

\section{References}

Abdrakhmanova A, Zwicker K, Kerscher S, Zickermann V, Brandt U (2006) Tight binding of NADPH to the $39-\mathrm{kDa}$ subunit of complex I is not required for catalytic activity but stabilizes the multiprotein complex. Biochim Biophys Acta 1757:1676-1682

Albracht SPJ (1974) Some new paramagnetic centers in submitochondrial particles detectable by EPR spectroscopy. Biochim Biophys Acta 347:183-192

Albracht SPJ (1980) The prosthetic groups in succinate dehydrogenase. Number and stoichiometry. Biochim Biophys Acta 612:11-28

Albracht SPJ (1982) In: Massey V, Williams CH (eds), Flavins and Flavoproteins: a possible solution to some unexplained observations on NADH dehydrogenase from beef-heart mitochondria. Elsevier/North-Holland Inc., Amsterdam, pp 759-762

Albracht SPJ (1984) In: Lee CP (eds), Current Topics in Bioenergetics: Applications of Electron Paramagnetic Resonance in the study of iron-sulfur clusters in energy-transducing membranes. Academic Press, New York, pp 79-106

Albracht SPJ (2010) The reaction of NADPH with bovine mitochondrial NADH:ubiquinone oxidoreductase revisited. II. Comparison of the proposed working hypothesis with literature data. J Bioener Biomem Accompanying paper. doi:10.1007/s10863-010-9302-y

Albracht SPJ, Subramanian J (1977) The number of Fe atoms in the iron-sulphur centers of the respiratory chain. Biochim Biophys Acta 462:36-48

Albracht SPJ, Bakker PT (1986) Evidence for two independent pathways of electron transfer in mitochondrial NADH:Q oxidoreductase. II. Kinetics of reoxidation of the reduced enzyme. Biochim Biophys Acta 850:423-428

Albracht SPJ, De Jong AMP (1997) Bovine-heart NADH:ubiquinone oxidoreductase is a monomer with $8 \mathrm{Fe}-\mathrm{S}$ clusters and 2 FMN groups. Biochim Biophys Acta 1318:92-106

Albracht SPJ, Hedderich R (2000) Learning from hydrogenases: location of a proton pump and of a second FMN in bovine NADHubiquinone oxidoreductase (Complex I). FEBS Lett 485:1-6

Albracht SPJ, Dooijewaard G, Leeuwerik FJ, Swol BV (1977) EPR signals of NADH: Q oxidoreductase. Shape and intensity. Biochim Biophys Acta 459:300-317

Albracht SPJ, Leeuwerik FJ, Van Swol B (1979) The stoichiometry of the iron-sulphur clusters $1 \mathrm{a}, 1 \mathrm{~b}$ and 2 of NADH:Q oxidoreductase as present in beef-heart submitochondrial particles. FEBS Lett 104:197-200

Albracht SPJ, Van Verseveld HW, Hagen WR, Kalkman ML (1980) A comparison of the respiratory chain in particles from Paracoccus denitrificans and bovine heart mitochondria by EPR spectroscopy. Biochim Biophys Acta 593:173-186

Albracht SPJ, Van der Linden E, Faber BW (2003) Quantitative amino acid analysis of bovine NADH:ubiquinone oxidoreductase (Complex I) and related enzymes. Consequences for the number of prosthetic groups. Biochim Biophys Acta 1557:41-49

Bakker PT, Albracht SPJ (1986) Evidence for two independent pathways of electron transfer in mitochondrial NADH:Q oxidoreductase. I. Pre-steady-state kinetics with NADPH. Biochim Biophys Acta 850:413-422

Beechey RB, Holloway CT, Knight IG, Roberton AM (1966) Dicyclohexylcarbodiimide - An inhibitor of oxidative phosphorylation. Biochem Biophys Res Commun 23:75-80 
Beechey RB, Roberton AM, Holloway CT, Knight IG (1967) The properties of dicyclohexylcarbodiimide as an inhibitor of oxidative phosphorylation. Biochemistry 6:3867-3879

Beinert H, Albracht SPJ (1982) New insights, ideas and unanswered questions concerning iron-sulfur clusters in mitochondria. Biochim Biophys Acta 683:245-277

Beinert H, Palmer G, Cremona T, Singer TP (1965) Kinetic studies on reduced diphosphopyridine nucleotide dehydrogenase by Electron Paramagnetic Resonance spectroscopy. J Biol Chem 240:475-480

Belogrudov G, Hatefi Y (1994) Catalytic sector of complex I (NADH: ubiquinone oxidoreductase): subunit stoichiometry and substrateinduced conformation changes. Biochemistry 33:4571-4576

Belogrudov GI, Hatefi Y (1996) Intersubunit interactions in the bovine mitochondrial complex I as revealed by ligand blotting. Biochem Biophys Res Commun 227:135-139

Berrisford JM, Sazanov LA (2009) Structural basis for the mechanism of respiratory complex I. J Biol Chem 284:29773-29783

Beyer RE, Brink TW, Crankshaw DL, Kuner JM, Pasternak A (1972) Effect of N, N'-dicyclohexylcarbodiimide and other carbodiimides on electron transfer catalyzed by submitochondrial particles. Biochemistry 11:961-969

Bridges HR, Grgic L, Harbour M, Hirst J (2009) The respiratory complexes I from the mitochondria of two Pichia species. Biochem J 422:151-159

Burgdorf T, Van der Linden E, Bernhard M, Yin QY, Back JW, Hartog AF, Muijsers AO, De Koster CG, Albracht SPJ, Friedrich B (2005) The soluble $\mathrm{NAD}^{+}$-reducing [NiFe]-hydrogenase from Ralstonia eutropha H16 consists of six subunits and can be specifically activated by NADPH. J Bacteriol 187:3122-3132

Carroll J, Fearnley IM, Skehel JM, Shannon RJ, Hirst J, Walker JE (2006) Bovine complex I is a complex of 45 different subunits. J Biol Chem 281:32724-32727

Chevallet M, Dupuis A, Issartel JP, Lunardi J, Van Belzen R, Albracht SPJ (2003) Two EPR-detectable [4Fe-4S] clusters, N2a and N2b, are bound to the NuoI (TYKY) subunit of NADH:ubiquinone oxidoreductase (Complex I) from Rhodobacter capsulatus. Biochim Biophys Acta 1557:51-66

Clason T, Ruiz T, Schägger H, Peng G, Zickermann V, Brandt U, Michel H, Radermacher M (2010) The structure of eukaryotic and prokaryotic complex I. J Struct Biol 169:81-88

Cleland KW, Slater EC (1953) Respiratory granules of heart muscle. Biochem J 53:547-556

Cremona T, Kearney EB (1964) Studies on the respiratory chainlinked NADH dehydrogenase. VI. Further purification and properties of the enzyme from beef heart. J Biol Chem 239:2328-2334

Darrouzet E, Issartel JP, Lunardi J, Dupuis A (1998) The 49-kDa subunit of NADH-ubiquinone oxidoreductase (Complex I) is involved in the binding of piericidin and rotenone, two quinonerelated inhibitors. FEBS Lett 431:34-38

De Jong AMP, Albracht SPJ (1994) Ubisemiquinones as obligatory intermediates in the electron transfer from NADH to ubiquinone. Eur J Biochem 222:975-982

De Jong AMP, Kotlyar AB, Albracht SPJ (1994) Energy-induced structural changes in NADH:Q oxidoreductase of the mitochondrial respiratory chain. Biochim Biophys Acta 1186:163-171

Djafarzadeh R, Kerscher S, Zwicker K, Radermacher M, Lindahl M, Schägger H, Brandt U (2000) Biophysical and structural characterization of proton-translocating NADH-dehydrogenase (complex I) from the strictly aerobic yeast Yarrowia lipolytica. Biochim Biophys Acta 1459:230-238

Djavadi-Ohaniance L, Hatefi H (1975) Oxidation of NADPH by submitochondrial particles from beef heart in complete absence of transhydrogenase activity from NADPH to NAD. J Biol Chem 250:9397-9403
Dooijewaard G, Slater EC (1976) Steady-state kinetics of high molecular weight (type-I) NADH dehydrogenase. Biochim Biophys Acta 440:1-15

Earley FGP, Ragan CI (1984) Photoaffinity labelling of mitochondrial $\mathrm{NADH}$ dehydrogenase with arylazidoamorphigenin, an analogue of rotenone. Biochem J 224:525-534

Earley FGP, Patel SD, Ragan CI, Attardi G (1987) Photolabelling of a mitochondrially encoded subunit of NADH dehydrogenase with $\left[{ }^{3} \mathrm{H}\right]$ dihydrorotenone. FEBS Lett 219:108-113

Fearnley IM, Walker JE (1992) Conservation of sequences of subunits of mitochondrial complex I and their relationships with other proteins. Biochim Biophys Acta 1140:105-134

Finel M, Majander AS, Tyynelä J, De Jong AMP, Albracht SPJ, Wikström M (1994) Isolation and characterisation of subcomplexes of the mitochondrial NADH:ubiquinone oxidoreductase (complex I). Eur J Biochem 226:237-242

Fontecilla-Camps JC, Volbeda A, Cavazza C, Nicolet Y (2007) Structure/function relationships of [NiFe]- and [FeFe]-hydrogenases. Chem Rev 107:4273-4303

Friedrich T (2001) Complex I: a chimaera of a redox and conformationdriven proton pump? J Bioenerg Bioemembr 33:169-177

Galante YM, Hatefi Y (1979) Purification and molecular and enzymic properties of mitochondrial NADH dehydrogenase. Arch Biochem Biophys 192:559-568

Galkin AS, Grivennikova VG, Vinogradov AD (1999) $\mathrm{H}^{+} / 2 \mathrm{e}^{-}$stoichiometry in NADH-quinone reductase reactions catalyzed by bovine heart submitochondrial particles. FEBS Lett 451:157-161

Gornall AG, Bardawill CJ, David MM (1949) Deterermination of serum proteins by means of the biuret reaction. J Biol Chem 177:755-766

Grigorieff N (1998) Three-dimensional structure of bovine NADH: ubiquinone oxidoreductase (complex I) at $22 \AA$ in ice. J Mol Biol 277:1033-1046

Guénebaut V, Vincentelli R, Mills D, Weiss H, Leonard KR (1997) Three-dimensional structure of NADH-dehydrogenase from Neurospora crassa by electron microscopy and conical tilt reconstruction. J Mol Biol 265:409-418

Guénebaut V, Schlitt A, Weiss H, Leonard K, Friedrich T (1998) Consistent structure between bacterial and mitochondrial NADH: ubiquinone oxidoreductase (Complex I). J Mol Biol 276:105-112

Gutman M, Singer TP, Casida JE (1970) Studies on the respiratory chain-linked reduced nicotinamide adenine dinucleotide dehydrogenase. XVII. Reaction sites of piericidin A and rotenone. J Biol Chem 245:1992-1997

Hatefi Y (1968) Flavoproteins of the electron transport system and the site of action of amytal, rotenone, and piericidin A. Proc Natl Acad Sci USA 60:733-740

Hatefi Y (1973) Oxidation of reduced triphosphopyridine nucelotide by sbmitochondrial particles from beef heart. Biochem Biophys Res Commun 50:978-984

Hatefi Y, Bearden AJ (1976) Electron paramagnetic resonance studies on the reduction of the components of complex I and transhydrogenase-inhibited complex I by NADH and NADPH. Biochem Biophys Res Commun 69:1032-1038

Hatefi Y, Hanstein WG (1973) Interactions of reduced and oxidized triphosphopyridine nucleotides with the electron-transport system of bovine heart mitochondria. Biochemistry 12:3515-3522

Hatefi Y, Stempel KE (1967) Resolution of complex I (DPNHcoenzyme Q reductase) of the mitochondrial electron transfer system. Biochem Biophys Res Commun 26:301-308

Hatefi Y, Stempel KE (1969) Isolation and enzymatic properties of the mitochondrial reduced diphosphopyridine nucleotide dehydrogenase. J Biol Chem 244:2350-2357

Hatefi Y, Yamaguchi M (1996) Nicotinamide nucleotide transhydrogenase: a model for utilization of substrate binding energy for proton translocation. FASEB J 10:444-452 
Hatefi Y, Haavik AG, Griffiths DE (1962) Studies on the electron transfer system; XL. Preparation and properties of mitochondrial DPNH-Coenzyme Q reductase. J Biol Chem 237:1667-1680

Hearshen DO, Dunham WR, Albracht SPJ, Ohnishi T, Beinert H (1981) EPR spectral stimulation on cluster N-1b in NADHubiquinone oxidoreductase of bovine heart mitochondria. FEBS Lett 133:287-290

Hinchliffe P, Sazanov LA (2005) Organization of iron-sulfur clusters in respiratory Complex I. Science 309:771-774

Hirst J, Carroll J, Fearnley IM, Shannon RJ, Walker JE (2003) The nuclear encoded subunits of complex I from bovine heart mitochondria. Biochim Biophys Acta 1604:135-150

Hofhaus G, Weiss H, Leonard K (1991) Electron microscopic analysis of the peripheral and membrane parts of mitochondrial NADH dehydrogenase (complex I). J Mol Biol 221:1027-1043

Kotlyar AB, Vinogradov AD (1990) Slow active/inactive transition of the mitochondrial NADH-ubiquinone reductase. Biochim Biophys Acta 1019:151-158

Kowal AT, Morningstar JE, Johnson MK, Ramsay RR, Singer TP (1986) Spectroscopic characterization of the number and type of iron-sulfur clusters in NADH:ubiquinone oxidoreductase. J Biol Chem 261:9239-9245

Krishnamoorthy G, Hinkle PC (1988) Studies on the electron transfer pathway, topography of iron-sulfur centers, and site of coupling in NADH-Q oxidoreductase. J Biol Chem 263:17566-17575

Lenaz G (1998) Quinone specificity of Complex I. Biochim Biophys Acta 1364:207-221

Lenaz G, Daves GD Jr, Folkers K (1968) Organice structural specificity and sites of Coenzyme $Q$ in succinoxidase and DPNH-oxidase systems. Arch Biochem Biophys 123:539-550

Lenaz G, Castelli A, Littarru GP, Bertoli E, Folkers K (1971) Specificity of lipids and Coenzyme Q in mitochondrial NADH and succin-oxidase of beef heart and S. cerevisiae. Arch Biochem Biophys 142:407-416

Leonard K, Haiker H, Weiss H (1987) Three-dimensional structure of NADH: ubiquinone reductase (complex I) from Neurospora mitochondria determined by electron microscopy of membrane crystals. J Mol Biol 194:277-286

Löw H, Vallin I (1963) Succinate-linked disphosphopyridine nucleotide reduction in submitochondrial particles. Biochim Biophys Acta 69:361-374

Lusty CJ, Machinist JM, Singer TP (1965) Studies on the respiratory chain-linked NADH dehydrogenase. VII; Labile sulfide groups in the dehydrogenase and in related proteins. J Biol Chem 240:1804-1810

Minakami S, Schindler FJ, Estabrook RW (1964) Hydrogen transfer between reduced diphosphopyridine nucleotide dehydrogenase and the respiratory chain. II. An initial lag in the oxidation of reduced diphosphopyridine nucleotide. J Biol Chem 239:2049-2054

Morgan DJ, Sazanov LA (2008) Three-dimensional structure of respiratory complex I from Escherichia coli in ice in the presence of nucleotides. Biochim Biophys Acta 1777:711-718

Murai M, Ishihara A, Nishioka T, Yagi T, Miyoshi H (2007) The ND1 subunit constructs the inhibitor binding domain in bovine heart mitochondrial complex I. Biochemistry 46:6409-6416

Ohnishi T (1975) Thermodynamic and EPR characterization of ironsulfur centers in the NADH-ubiquinone segment of the mitochondrial respiratory chain in pigeon heart. Biochim Biophys Acta 387:475-490

Ohnishi T (1979) In: Capaldi RA (eds), Membrane Proteins in Energy Transduction: mitochondrial iron-sulfur flavodehydrogenases. Marcel Dekker, New York, pp 1-87

Ohnishi T (1998) Iron-sulfur clusters/semiquinones in Complex I. Biochim Biophys Acta 1364:186-206

Ohnishi T, Blum H, Galante YM, Hatefi Y (1981) Iron-sulfur N-1 clusters studied in NADH-ubiquinone oxidoreductase and in soluble NADH dehydrogenase. J Biol Chem 256:9216-9220
Orme-Johnson NR, Orme-Johnson WH, Hansen RE, Beinert H, Hatefi Y (1971) EPR detectable electron acceptors in submitochondrial particles from beef heart with specific reference to the iron-sulfur components of DPNH-ubiquinone reductase. Biochem Biophys Res Commun 44:446-452

Orme-Johnson NR, Hansen RE, Beinert H (1974a) Electron paramagnetic resonance-detectable electron acceptors in beef heart mitochondria. Reduced diphosphopyridine nucleotide ubiquinone reductase segment of the electron transfer system. J Biol Chem 249:1922-1927

Orme-Johnson NR, Hansen RE, Beinert H (1974b) Electron paramagnetic resonance-detectable electron acceptors in beef heart mitochondria. Ubihydroquinone-cytochrome c reductase segment of the electron transfer system and complex mitochondrial fragments. J Biol Chem 249:1928-1939

Paech C, Reynolds JR, Singer TP, Holm RH (1981) Structural identification of the iron-sulfur clusters of the respiratory chainlinked NADH dehydrogenase. J Biol Chem 256:3167-3170

Papa S, De Rasmo D, Scacco S, Signorile A, Technikova-Dobrova Z, Palmisano G, Sardanelli AM, Papa F, Panelli D, Scaringi R, Santeramo A (2008) Mammalian complex I: a regulable and vulnerable pacemaker in mitochondrial respiratory function. Biochim Biophys Acta 1777:719-728

Peng G, Fritzsch G, Zickermann V, Schagger H, Mentele R, Lottspeich F, Bostina M, Radermacher M, Huber R, Stetter KO, Michel H (2003) Isolation, characterization and electron microscopic single particle analysis of the NADH:ubiquinone oxidoreductase (complex I) from the hyperthermophilic eubacterium Aquifex aeolicus. Biochemistry 42:3032-3039

Pilkington SJ, Skehel JM, Gennis RB, Walker JE (1991) Relationship between mitochondrial NADH-ubiquinone reductase and a bacterial NAD-reducing hydrogenase. Biochemistry 30:21662175

Prieur I, Lunardi J, Dupuis A (2001) Evidence for a quinone binding site close to the interface between NUOD and NUOB subunits of Complex I. Biochim Biophys Acta 1504:173-178

Radermacher M, Ruiz T, Clason T, Benjamin S, Brandt $\mathrm{U}$, Zickermann V (2006) The three-dimensional structure of complex I from Yarrowia lipolytica: a highly dynamic enzyme. J Struct Biol 154:269-279

Ragan CI (1976) The interaction of reduced nicotinamide-adenine dinucleotide phosphate with reduced nicotinamide-adenine dinucleotide-ubiquinone reductase from bovine heart mitochondria. Biochem J 158:149-151

Ragan CI, Widger WR, King TE (1974) Pyridine nucleotide transhydrogenase activity of soluble cardiac NADH dehydrogenase and particulate NADH-ubiquinone reductase. Biochem Biophys Res Commun 60:894-900

Reda T, Barker CD, Hirst J (2008) Reduction of the iron sulfur clusters in mitochondrial NADH:ubiquinone oxidoreductase (complex I) by $\mathrm{Eu}^{\mathrm{II}}$-DTPA, a very low potential reductant. Biochemistry 47:8885-8893

Ringler RL, Minakami S, Singer TP (1960) Isolation and properties of the DPNH dehydrogenase of the respiratory chain from heart mitochondria. Biochem Biophys Res Commun 3:417-422

Ringler RL, Minakami S, Singer TP (1963) Studies on the respiratory chain-linked NADH dehydrogenase. II. Isolation and molecular properties of the enzyme from beef heart. J Biol Chem 238:801810

Rydström J, Teixeira da Cruz A, Ernster L (1971) Steady-state kinetics of mitochondrial nicotinamide nucleotide transhydrogenase. 2. The energy-linked reaction. Eur J Biochem 23:213-219

Rydström J, Montelius J, Bäckström D, Ernster L (1978) The mechanism of oxidation of reduced nicotinamide dinucleotide phosphate by submitochondrial particles from beef heart. Biochim Biophys Acta 501:370-380 
Sazanov LA, Hinchliffe P (2006) Structure of the hydrophilic domain of respiratory Complex I from Thermus thermophilus. Science 311:1430-1436

Schulte U, Haupt V, Abelmann A, Fecke W, Brors B, Rasmussen T, Friedrich T, Weiss H (1999) A reductase/isomerase subunit of mitochondrial NADH:ubiquinone oxidoreductase (complex I) carries an NADPH and is involved in the biogenesis of the complex. J Mol Biol 292:569-580

Singer TP (1979) Mitochondrial electron-transport inhibitors. Meth Enzymol 55:454-462

Van Belzen R (1991) Electron transfer in NADH:Q oxidoreductase from bovine-heart mitochondria. Ph.D. Thesis, University of Amsterdam, Amsterdam, The Netherlands

Van Belzen R, Albracht SPJ (1989) The pathway of electron transfer in NADH:Q oxidoreductase. Biochim Biophys Acta 974:311-320

Van Belzen R, Van Gaalen MC, Cuypers PA, Albracht SPJ (1990) New evidence for the dimeric nature of NADH:Q oxidoreductase in bovine-heart submitochondrial particles. Biochim Biophys Acta 1017:152-159

Van Belzen R, De Jong AMP, Albracht SPJ (1992) On the stoichiometry of the iron-sulphur clusters in mitochondrial NADH:ubiquinone oxidoreductase. Eur J Biochem 209:1019-1022

Van Belzen R, Kotlyar AB, Moon N, Dunham WR, Albracht SPJ (1997) The iron-sulfur clusters 2 and ubisemiquinone radicals of NADH:ubiquinone oxidoreductase are involved in energy coupling in submitochondrial particles. Biochemistry 36:886-893

Van der Linden E, Faber BW, Bleijlevens B, Burgdorf T, Bernhard M, Friedrich B, Albracht SPJ (2004) Selective release and function of one of the two FMN groups in the cytoplasmic $\mathrm{NAD}^{+}$reducing [NiFe]-hydrogenase from Ralstonia eutropha. Eur $\mathrm{J}$ Biochem 271:801-808

Vanneste WH (1966) Molecular proportion of the fixed cytochrome components of the respiratory chain of Keilin-Hartree particles and beef heart mitochondria. Biochim Biophys Acta 113:175-178

Volbeda A, Charon MH, Piras C, Hatchikian EC, Frey M, FontecillaCamps JC (1995) Crystal structure of the nickel-iron hydrogenase from Desulfovibrio gigas. Nature 373:580-587

Volbeda A, Garcia E, Piras C, De Lacey AL, Fernandez VM, Hatchikian EC, Frey M, Fontecilla-Camps JC (1996) Structure of the [NiFe] hydrogenase active site: Evidence for biologically uncommon Fe ligands. J Am Chem Soc 118:12989-12996

Walker JE (1992) The NADH:ubiquinone oxidoreductase (complex I) of respiratory chains. Q Rev Biophys 25:253-324
Walker JE, Arizmendi JM, Dupuis A, Fearnley IM, Finel M, Medd SM, Pilkington SJ, Runswick MJ, Skehel JM (1992) Sequences of 20 subunits of NADH:ubiquinone oxidoreductase from bovine heart mitochondria. Application of a novel strategy for sequencing proteins using the polymerase chain reaction. J Mol Biol 226:1051-1072

Wikström M (1984) Two protons are pumped from the mitochondrial matrix per electron transferred between NADH and ubiquinone. FEBS Lett 169:300-304

Yagi T (1987) Inhibition of NADH-ubiquinone reductase activity by $\mathrm{N}, \mathrm{N}^{\prime}$ - dicyclohexylcarbodiimide and correlation of this inhibition with the occurrence of energy-coupling site 1 in various organisms. Biochemistry 26:2822-2828

Yagi T, Hatefi Y (1988) Identification of the dicyclohexylcarbodiimidebinding subunit of NADH- ubiquinone oxidoreductase (Complex I). J Biol Chem 263:16150-16155

Yagi T, Matsuno-Yagi A (2003) The Proton-Translocating NADHQuinone Oxidoreductase in the Respiratory Chain: The Secret Unlocked. Biochemistry 42:2266-2274

Yakovlev G, Reda T, Hirst J (2007) Reevaluating the relationship between EPR spectra and enzyme structure for the iron-sulfur clusters in NADH:quinone oxidoreductase. Proc Natl Acad Sci USA 104:1270-12725

Yamaguchi M, Belogrudov GI, Hatefi Y (1998) Mitochondrial NADH-ubiquinone oxidoreductase (Complex I). Effect of substrates on the fragmentation of subunits by trypsin. J Biol Chem 273:8094-8098

Yamaguchi M, Belogrudov GI, Matsuno-Yagi A, Hatefi Y (2000) The multiple nicotinamide nucleotide-binding subunits of bovine heart mitochondrial NADH:ubiquinone oxidoreductase (complex I). Eur J Biochem 267:329-336

Yano T, Sklar J, Nakamaru-Ogiso E, Takahashi Y, Yagi T, Ohnishi T (2003) Characterization of cluster N5 as a fast-relaxing [4Fe-4S] cluster in the Nqo3 subunit of the proton-translocating NADHubiquinone oxidoreductase from Paracoccus denitrificans. J Biol Chem 278:15514-15522

Yano T, Dunham WH, Ohnishi T (2005) Characterization of the $\Delta \mu_{\mathrm{H}}+$-sensitive ubisemiquinone species $\left(\mathrm{SQ}_{\mathrm{Nf}}\right)$ and the interaction with cluster N2: new insight into the energy-coupled electron transfer in Complex I. Biochemistry 44:1744-1754

Zickermann V, Kerscher S, Zwicker K, Tocilescu MA, Radermacher M, Brandt U (2009) Architecture of complex I and its implications for electron transfer and proton pumping. Biochim Biophys Acta 1787:574-583 